\title{
An Interactive Preliminary Design System of High Speed Forebody and Inlet Flows
}

\author{
May-Fun Liou ${ }^{1}$ Thomas J. Benson. ${ }^{2}$ and Charles J. Trefny ${ }^{3}$ \\ NASA Glenn Research Center, Cleveland, OH 44135, U.S.A.
}

\begin{abstract}
This paper demonstrates a simulation-based aerodynamic design process of high speed inlet. A genetic algorithm is integrated into the design process to facilitate the single objective optimization. The objective function is the total pressure recovery and is obtained by using a PNS solver for its computing efficiency. The system developed uses existing software of geometry definition, mesh generation and CFD analysis. The process which produces increasingly desirable design in each genetic evolution over many generations is automatically carried out. A generic two-dimensional inlet is created as a showcase to demonstrate the capabilities of this tool. A parameterized study of geometric shape and size of the showcase is also presented.
\end{abstract}

\section{Introduction}

The art of high speed inlet design is a trade-off among various factors, including weight, aerodynamic performance and pressure recovery. The primary consideration of the efficiency of an inlet is the total pressure recovery which represents the available work that can be extracted from the captured mass flow. Losses of total pressure are due to the oblique shock system in the supersonic section of the inlet, the viscous boundary layer, the terminal normal shock and shock-boundary layer interaction. The pay-off of a high recovery inlet is substantial. Most gas turbine engines require the Mach number at the engine face at a moderate subsonic speed, typically around Mach 0.4. A preferable high static pressure at the compressor face has compromised the high recovery objective. In Hypersonic flight regime, although ramjet or scramjet depending on high speeds to compress the air without the help from compressor machinery, either has to combine with turbojet to make the flight across the range of speeds possible. High speed inlet design has become a problem involving multiple parameters and multiple objectives, not to mention the multiple disciplines involved. The data of total pressure recovery at engine face has a determining effect on engine thrust and aerodynamic evaluation of a whole aircraft. Therefore, a design tool is needed.

Due to the complexity of shock waves and boundary layers interaction, the use of the traditional techniques, like the method of characteristic or streamline tracing, becomes inadequate for preliminary design stage. Computational Fluid dynamics (CFD) has allowed engineers to test new designs quickly before carrying out experiments. Integrating CFD and other computer-aided simulation programs, a system has been developed for the preliminary design of high speed inlets and forebodies. A graphical user interfaces written in Matlab $\odot$ is provided to designer for an interactive design environment. Continuing the development ${ }^{1}$, two new elements are added to the system. First, a geometry controller, Capri, is included. CAPRI provides a simple control and easy access to the CAD models for simulation driven design. Because the inlet must provide optimum performance over a wide range of speed, a large matrix of geometric and flow variables are mandated to obtain an optimum configuration. Those can be very time consuming and computationally intensive. Working as a checking mechanism, secondly, an optimizer is included to aid engineers in making multiple objectives selections and compromises, for instance those of the compression methods, number of oblique shocks or ramps and the cross section shape of supersonic section. As a result, not only is high -speed calculation required, but also the automation in finding optimum solution is desired. The automation of the system has been implemented and is driven by an optimizer. The resulting Inlet Preliminary and Optimizing Design $\underline{S}$ ystem (IPODS) comprises six functions: geometry definition, geometry controller, flow grid generation, a Parabolized Navier-Stokes (PNS) flow solver, graphics post-processor and optimizer,

\footnotetext{
${ }^{1}$ Aerospace Engineer, Multidisciplinary Design, Analysis, and Optimization Branch, 21000 Brookpark Rd., MS 5-11, AIAA Member.

${ }^{2}$ Aerospace Engineer, Inlets and Nozzles Branch, 21000 Brookpark Rd., MS 5-11, AIAA Member.

${ }^{3}$ Aerospace Engineer, Inlets and Nozzles Branch, 21000 Brookpark Rd., MS 5-11, AIAA Senior Member.
} 
shown schematically in Figure 1. Matlab $\odot$ and its optimizing toolbox provide the required computing environment and drive the entire design process. Brief descriptions of each function are given in the following section. All computations can be carried out in serial or parallel computing environment.

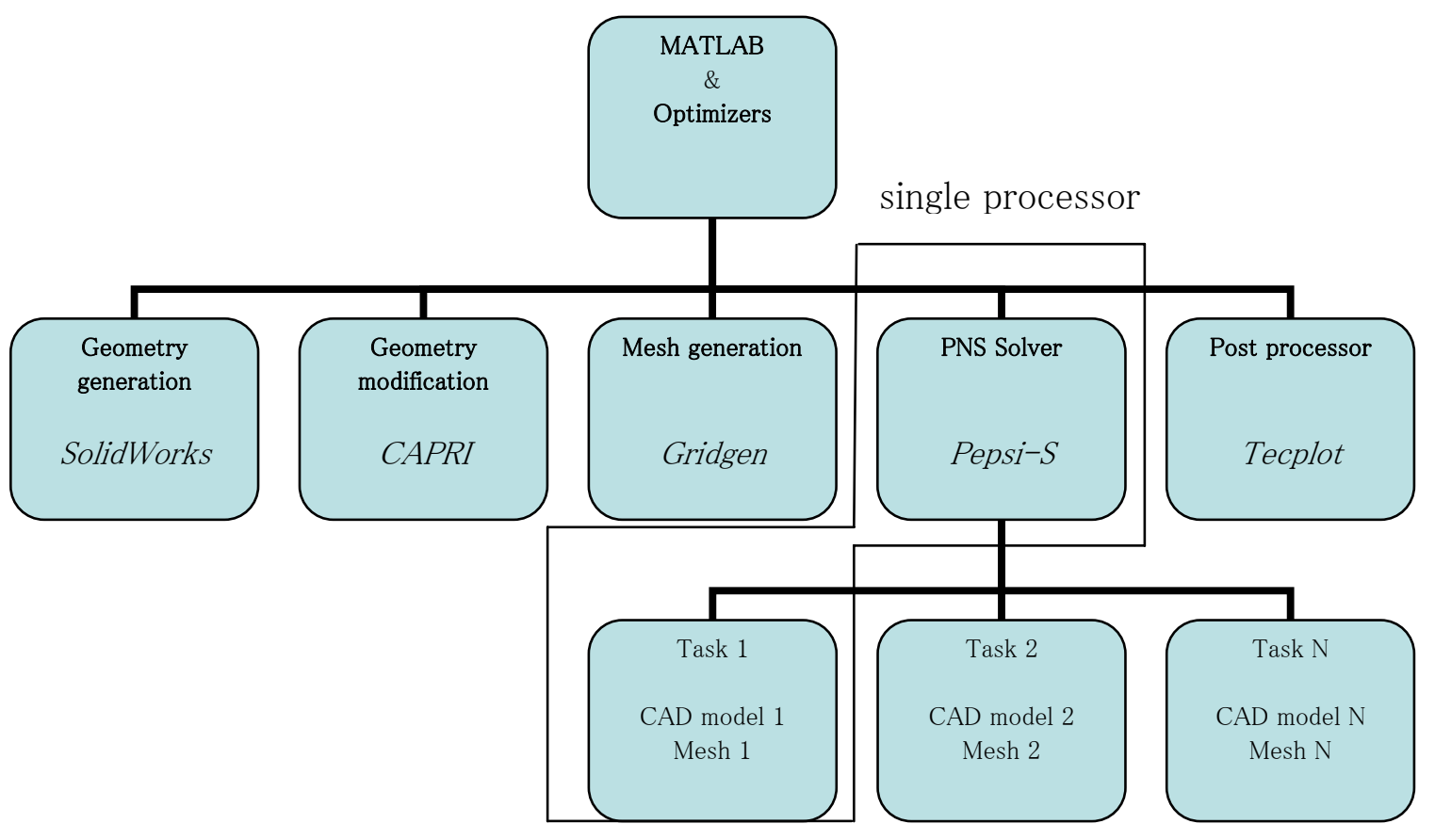

Figure 1: Schematic representation of a high speed inlet design framework.

\section{Geometry Definition}

With a future extension of this system to include propulsive system (pylon and nacelles) for multi-disciplinary analysis and optimization (MDAO) and interest in developing unconventional configuration in mind, a parametric CAD-based model is adopted. The emphasis on a central unified representation of the geometry for all disciplines considered has advantages over legacy approaches which use IGES, STEP, etc. A parametric model can contain the design intent, can be easily updated to a new instance, and the final result can be integrated with manufacture. In this framework, SolidWorks $(\mathrm{C}$ is used to build the parametric model of inlet. SolidWorks $\odot$ is widely used in industrial design community and uses Parasolid geometry kernel. An example of a three-dimensional inlet solid model done by SolidWorks is shown in Figure 2. The solid model which is extruded from a two dimensional, double-wedge geometry has design parameters of first and second ramp angles, inlet length and inlet height.

\section{Geometry Management}

CAPRI provides a vendor-neutral platform to assemble a multidisciplinary system which can be tightly coupled with CAD. It provides a translation-free connectivity to native CAD. The CAPRI CAE Gateway exports geometry to the formats of several commonly used preprocessor and meshing tools. Thus, grid generator can read in CAD geometry directly through CAPRI's tessellation, a tight coupling between CAD and analysis. Using the solid inlet part from SolidWorks $\bigcirc$ in Figure 2, Figure 3 shows an interactive geometry management through CAPRI GUI. In its Parameters and Features panes, CAPRI ${ }^{2}$ lists all engineering design intents from CAD file and allows user to modify the values of parameters interactively. After any changes, the new geometry is displayed accordingly. Figure 4 presents the MATLAB plots, from left to right, of the inlet ramp before and after a $10^{\circ}$ increase of the first wedge angle via CAPRI. 


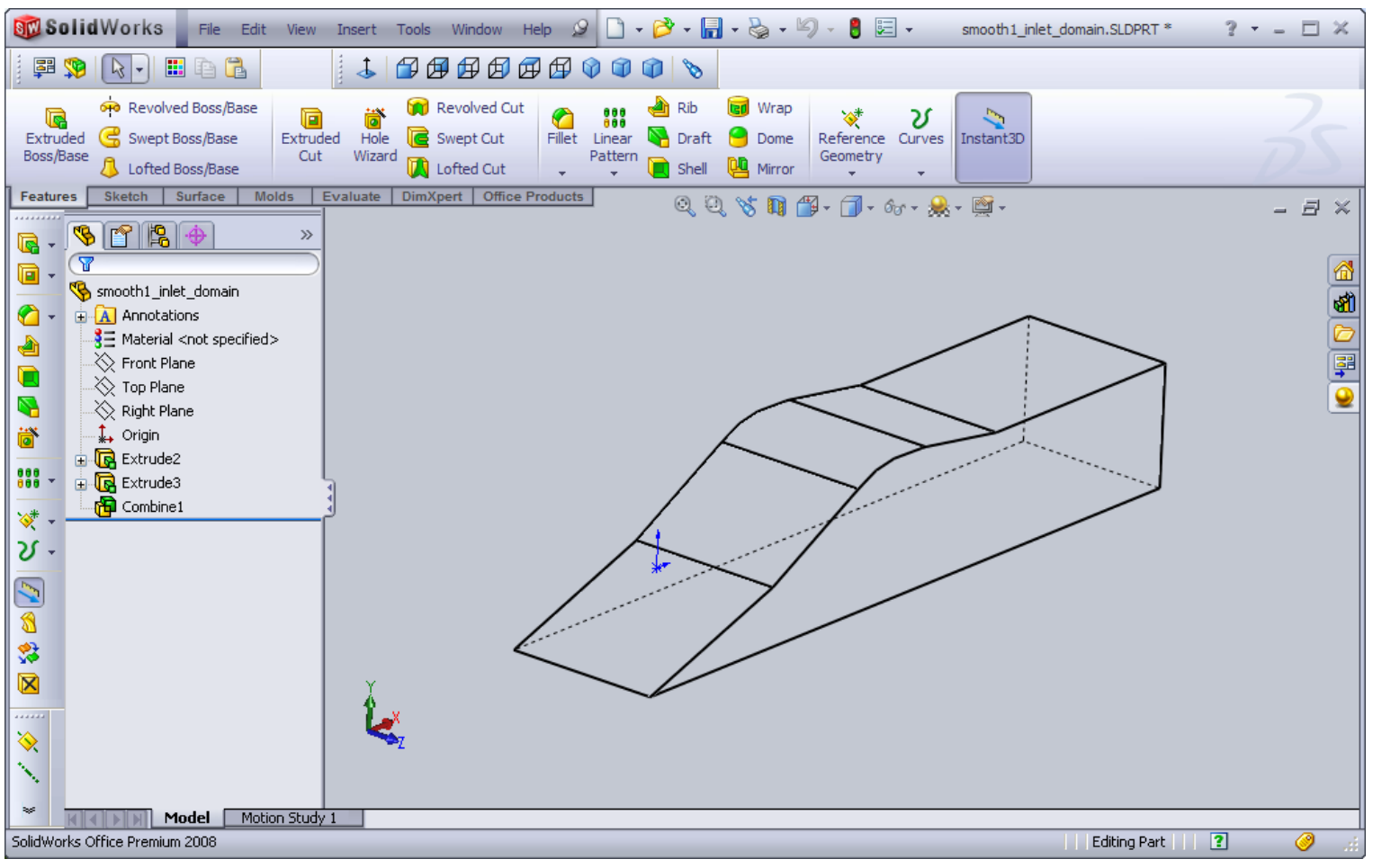

Figure 2: A 3D inlet part geometry, shown is a double-wedge center body, generated by SolidWorks $\odot$.

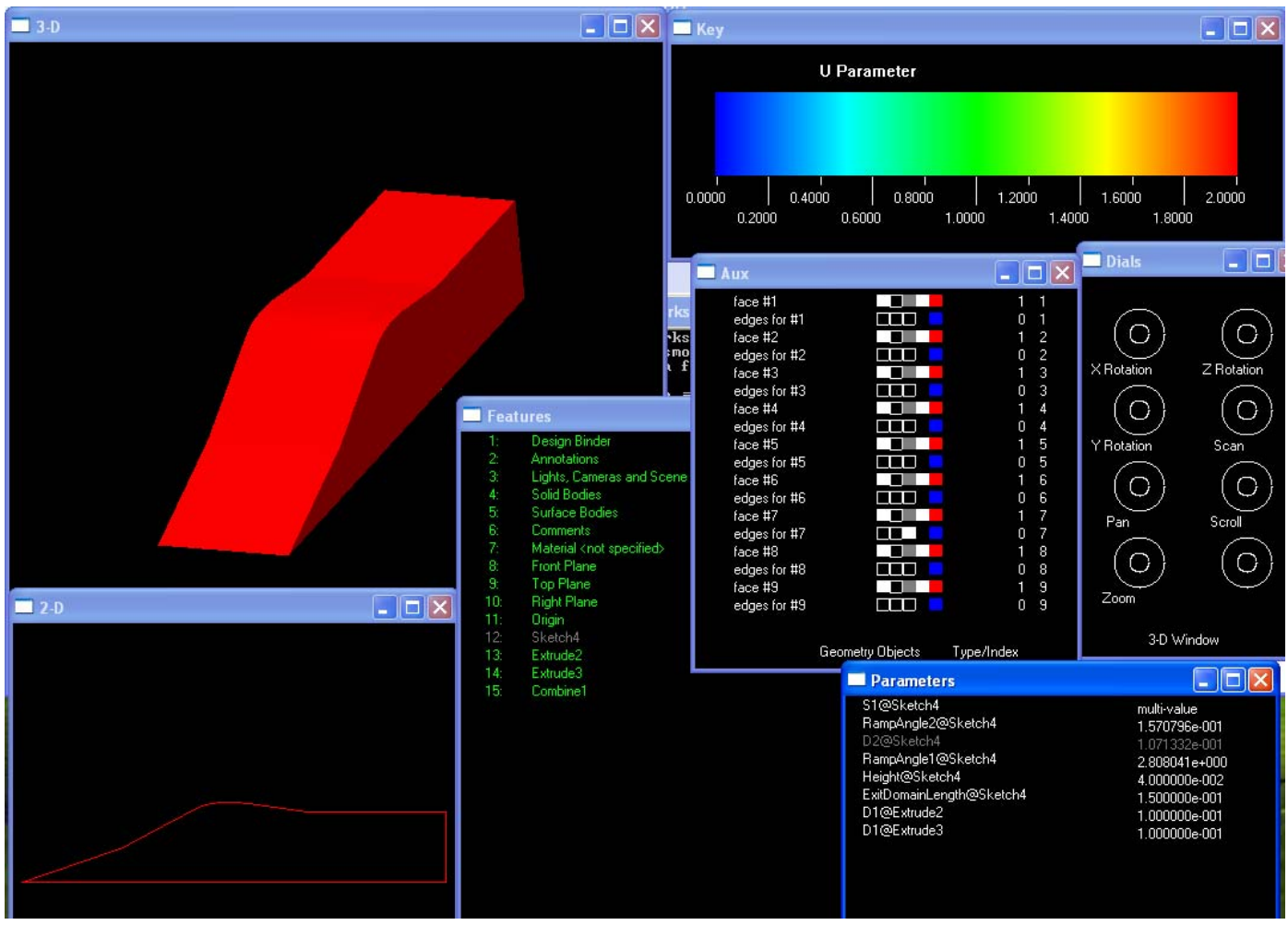

Figure 3: Geometry is display and managed interactively through CAPRI GUI. 


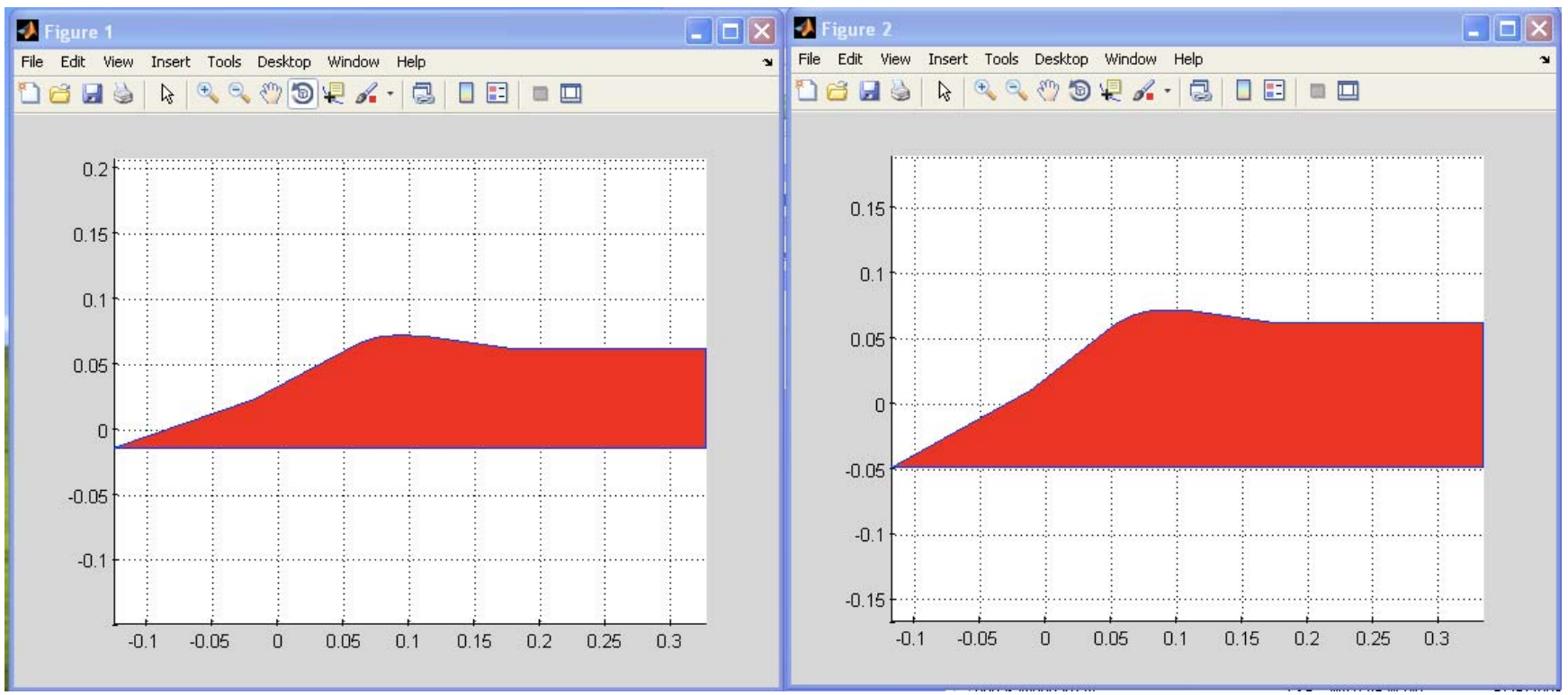

Figure 4: Parametric modification of inlet ramp angle (+10 degree). Update is performed in MATLAB via CAPRI.

\section{Grid Generation}

PEPSI-S requires a flow skeleton grid to define the surfaces of inlet. The skeleton grid is required to be orthogonal. A commercial grid generation, Gridgen $\odot$ and the Annular Diffuser Deck (ADD) $)^{3}$ are used to generate skeleton grids. Both can be executed by MATLAB GUI. To automate the mesh generation, user can use a Gridgen's scripting language, Glyph, to include design variables in its journal file. The glyph script can be easily edited to change the value of design variables or to refine mesh resolution. Then, the glyph file can be executed in batch mode to generate a new set of grid, details listed in Ref. 4. Also, the mesh update can be facilitated through Capri and Figure 5 presents the fluid (non-solid) part of the same geometry which is exported from CAPRI gateway to Gridgen and the resulting meshes.

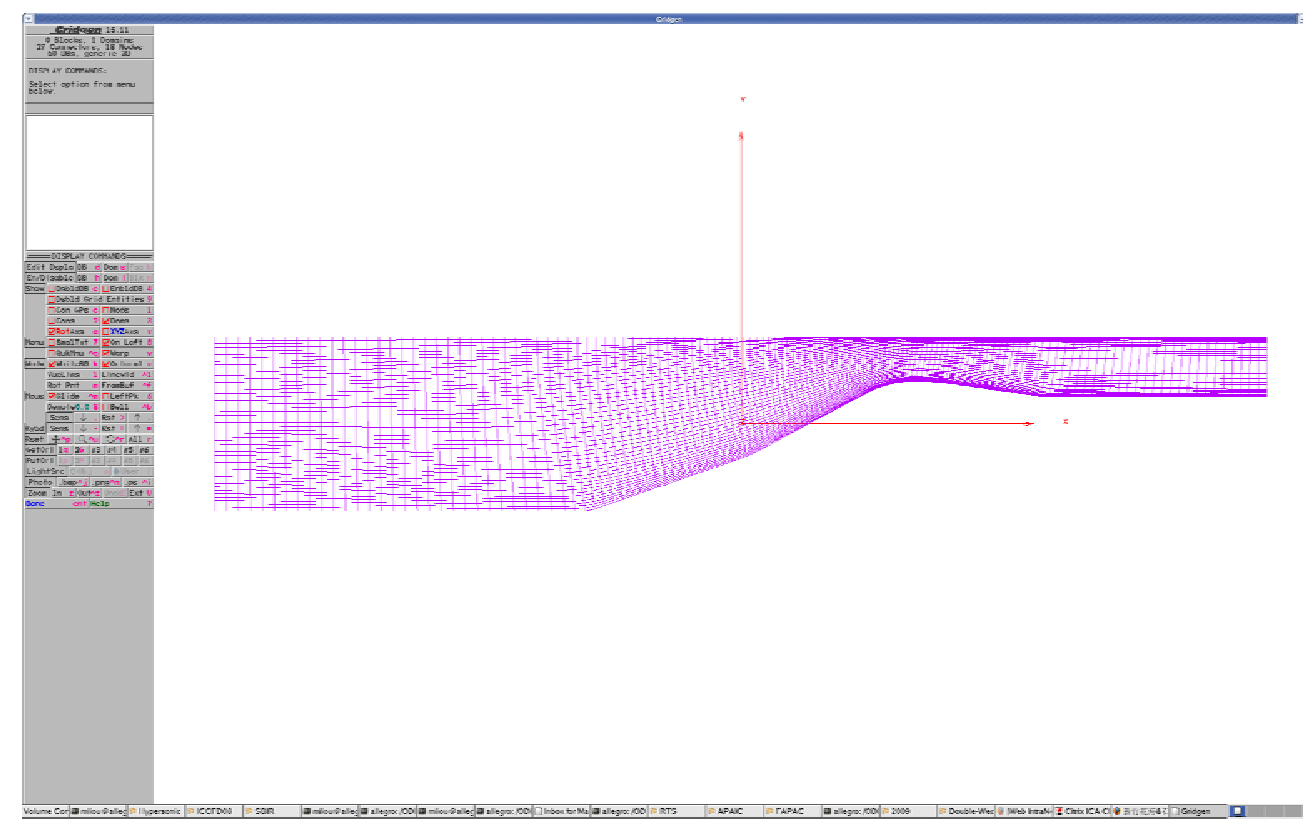

Figure 5: Mesh points at the center plane of the double wedge inlet.

\section{PNS solver}

At the center of this system is the PNS solver, PEPSI-S computer program. It is an acronym of Parabolic Elliptic Streamwise Implicit-Supersonic and has been verified for several hypersonic problems ${ }^{1}$. The solver uses a single pass, 
spatial marching technique to solver PNS equations for supersonic and hypersonic flow through a specified geometry. It is known that PNS can not model flow past a normal shock and it encounters serious convergence problems in large regions of subsonic and reverse flows, where the neglected stream-wise diffusion terms are important. But it provides detailed computational analysis on where to eliminate low speed flow and allows designer revising geometry accordingly. And the speed of PNS flow solver lands itself a good candidate for preliminary design, even in the phase of conceptual design. PEPSI-S includes boundary conditions of mass removal (sinks) and addition (sources) which are useful in modeling commonly used boundary layer bleed in inlet. It supports two-equation turbulence models for further development of improved boundary layer transition models for hypersonic applications. Currently PEPSI-S can calculate one million mesh points in two and half minutes as reported in Ref. 1.

In Figure 1, option between single processor, i.e. serial computing, and multiple processors, i.e. parallel computing depends on the computing resources available. In this paper, automation is carried out by single processor. It also provides two design modes: interactive and automated. Matlab $\odot$ and its optimizing toolbox provide the required computing environment and drive the entire design process. A set of Matlab@'s script files serve as utility functions that generate all necessary input files for each component. The flowchart of the system and a detailed description of its automation are given in Figure 6 and are covered in the next section.

The objective of this work is two folds. One is to facilitate the above mentioned design processes with an optimizer and to develop a streamlined, automated process in running all afore-mentioned computer programs. In this framework, user can choose to perform a design with engineer in the loop or using the optimizer. The other goal is to demonstrate how an optimizer can be used in obtaining the geometric optimum of high speed inlets. "Optimum" is defined as the maximum achievable total pressure recovery at the exit of inlet. To account for the non-uniform flow field at the exit caused by viscous effect, an area averaged total pressure recovery is used as objective function required by optimizer. Furthermore, validation of the system and parametric studies on optimizing two-dimensional, mixed compression, double-ramp high speed inlets are also included.

\section{Automating the design process}

Figure 6 shows the flow chart of the automating processes and the relationship among different functions. Inlet design space generally comprises inlet length, number of oblique shocks and their respective ramp/cone angles, position and geometric characters of cowl lip, allowable level of distortion, and the captured mass flow rate. Design variables can be any combination of variables in the design space, while the output, or so called objective function here is the total pressure recovery. An objective function is a function of design variables and provides the measurement of how well of a given design performs. In this study, distortion is not considered. Matlab $\odot$ script files generate all necessary input files for Capri@), Gridgen $($ ), Annular Diffuser Deck (ADD) code, PEPSI-S and the optimizer. There are different types of optimization schemes in MATLAB optimization toolbox, for instance, symmetric Latin hypercube, direct search, etc. ${ }^{9}$ Mimicking the biological evolution, genetic algorithms (GAs) search the global minimum which designer is more interested in. First, GA randomly generate an initial "population" of potential designs, in terms of sets of chosen design variables. Each set is also called individual. Each individual obtains a "fitness" value from the objective function. Individuals with higher fitness value are more likely to reproduce, so that their traits can be passed on to the next generation. Mutation and crossover are introduced to ensure diversity in population, so that the algorithm can consider more possibilities. For each individual, elements in the system are called to define geometry, change geometry, generate mesh, run solver, and evaluate fitness value. If there are twenty individuals and fifty generations are used, then there are one thousand calls to each element. The whole processes are very time consuming. In the current study, an automated process is developed for generating input files for mesh generator and solver, completing the analyses, and evaluating the value of objective functions. The entire process is driven by a set of Matlab script files. Gridgen or ADD journal files are generated manually only for the baseline inlet configuration. This journal file contains defined design variables and commands to construct points, edges, surface mesh, and volume mesh if three dimensional configuration is considered. Also, included in the journal file is the grid export format, which is plot3D here. Similarly, a template of PEPSI-S input file, including free stream conditions, boundary conditions etc., can be generated manually for solver by using its interactive mode. Those templates are used later to run grid generator and solver in automatic mode.

Although there is an inherent parallelism in GAs, a perfect fit for parallel processors, this advantage is not exploited in this study, which is completely restricted to a single processor operation. 


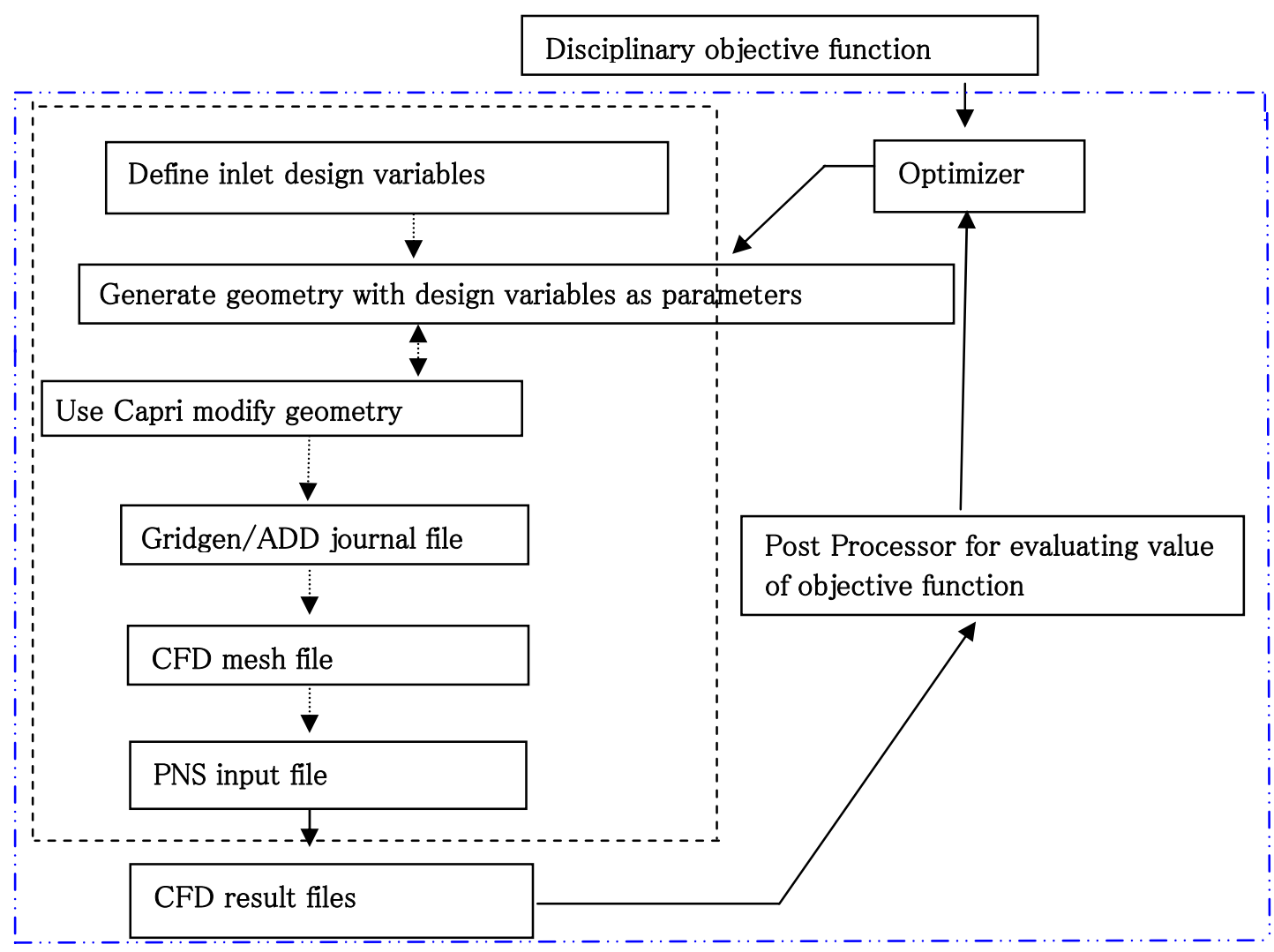

Figure 6: Flow chart of the automated process, indicated by blue dashed line, in creating optimized design from CAD to Computer Aided Analysis (CAA); the user intervention mode is shown in black dotted line.

\section{Problem Definition}

\section{Inlet Design}

Thus, tradeoff of geometric size of inlet, position and strength of shocks are critical in maximizing the total pressure recovery, the design criterion. In a two-dimensional high speed inlet, i.e. flight Mach number above 2, a mixed compression multi-ramp system is usually considered a preferable choice. To achieve a parametric study in design space, a baseline geometry selected here is a double ramp center body with a cowl. This geometry can be resulted from the trade-off among pressure recovery, flow complication, and inlet weight. Several set of design variables and their corresponding constraints are selected to demonstrate the capability of this development. Cases are built progressively to demonstrate how GA determines the optimum shape and size of two-dimensional inlets. First, a theoretical case is adopted to validate the chosen optimizer. Then, a generic two-dimensional inlet is used as a showcase to demonstrate the capabilities of this tool.

\section{Fitness}

As stated before, the goal of inlet design is to maximize the area averaged total pressure recovery at exit. For most optimizers, algorithms are designed to look for minimal function value. Therefore, the fitness function here is the deficiency of total pressure recovery from a perfect recovery. In another word, fitness value is $1-\left(\mathrm{Pt} / \mathrm{P}_{\infty}\right)$, where $\mathrm{P}_{\infty}$ is the free stream total pressure and $\mathrm{Pt}$ is the area averaged total pressure at the exit plane. Because those required parent selection, mutation, crossover, and elitism in GA are based on the fitness value obtained by running flow solver, a default value of 0.999 is given instead when solver fails. GA plots in the following sections are plots of the population distribution vs. generation, of the best and mean fitness value with respect to generation and the GA command panel. With increasing geometric complexity, there possibly are large gaps between the best and mean fitness values in some generations because solver fails more to converge than simple cases.

\section{GA setup}

The genetic algorithm uses individuals in the current generation to create the children that make up the next generation. There are three types of children: elite children, crossover children and mutation children. Elites are the fittest individuals and are guaranteed to survive to the next generation. The crossover children are reproduced by selecting genes or vector entries from a pair of individuals in the current generation and combining them. Mutation children are created by applying 
random changes to a single individual in the current generation. In the following cases, default settings are used if not specified. Those are: twenty individuals per generation, two elites going to the next generation, fourteen crossover children, and four mutation children. The default stopping conditions for the genetic algorithm are shown in its Stopping criteria pane in the optimization tool, shown later in plots of GA command panel, and is listed in Ref. 5.

\section{Test Cases: Validation of the GA}

In Oswatitsch (1944) best shock theory, for a system of n-1 oblique shocks occurring ahead of a normal shock in two dimensions, the maximum shock recovery is obtained if oblique shocks are of equal strength ${ }^{6}$. A schematic display of such system is shown in Figure 7. In the past, this optimization criterion has been used in various preliminary supersonic and hypersonic inlet design ${ }^{7,8}$, and is utilized here to validate the optimizer used in this study. Benson ${ }^{1}$ has developed a program based on NACA 1365 theoretical formulations to compute the inviscid shock waves. Instead of PEPSI-S, this inviscid program is integrated into the system and is used as the solver for a fair comparison with Oswatitsch's inviscid results.

To determine the ramp angles, $\delta_{1}$ and $\delta_{2}$ of a two-wedge ramp/inlet, for maximum pressure recovery on the supersonic section, the aforementioned GA solver of the MathWorks $\odot$ has been set up and been run for inflow Mach number from 1.5 to 5 . The results of each optimum total pressure recovery and its corresponding optimum angle combination of first and second wedges, $\left(\delta_{1}, \delta_{2}\right)$, are compared with those of Oswatitsch's theory and those found in Reference 7. Figures from 8 to 12 show the resulting MATLAB ${ }^{\circledR}$ plotting windows for inflow Mach number at 3 . Each figure displays a set of two plots. On the top, the best and mean values of the objective function are plotted against generation. And, the lower plot displays the distribution of the population at the current generation, with magenta colored lines representing the linear, nonlinear inequality and bound constraints, and with contours of the defined objective function. At the initial stage, see Figure 8, individuals are produced by random number generator and thus biased to lay in the domain between 0 and 1 for both wedge angle 1 and 2 . In the first generation, Figure 9 shows the best value has dropped to be less than one half of the initial one. In Figure 10, even with a moderate population size, 20, the high-scoring individual quickly took over the "gene" pool in the second generation. The optimal solution is obtained on the third generation, and evolution stops at the fifth generations after meeting the convergence criteria. The final population eventually concentrates around the minimum point as shown in Figure 11. The setup for the GA run is given in Figure 12, which is the screen dump of the GA command window.

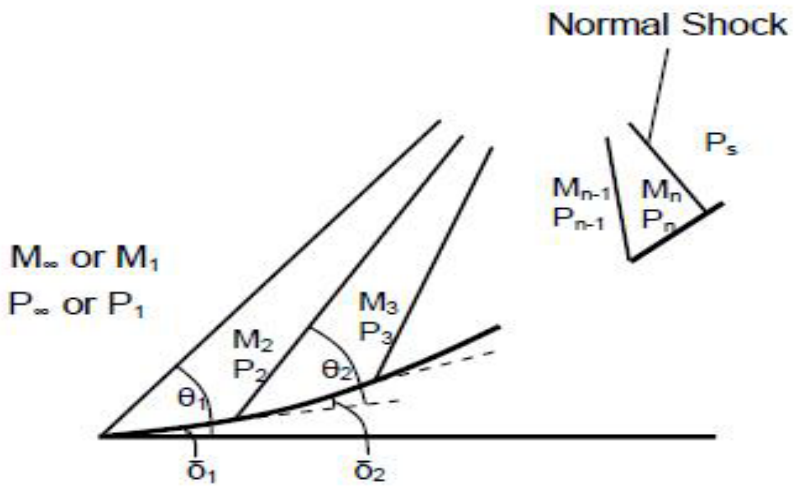

Figure 7: Multiple shock compression system for Oswatitsch shock theory.

In Matlab $\odot$ genetic algorithm (GA) solver, mutation and crossover are used to produce new individuals at every generation. Note that the GA solver provides a default mutation function named mutaiongaussian which is only appropriate for nonconstrained minimization problems. For the constrained minimization problems, mutation function is switched to another function called mutationadaptfeasible to ensure the converged solution would satisfy constraints, which may be nonlinear or linear functions of design variables. To explore those aforementioned features, a study of effects of imposing constraints or without constraints is presented. In the double-wedge inlet case, Figure 13 shows the optimized solution after running GA without constraints. It takes a much longer evolutions to converge because individuals are unconstrained but bounded (both angles have to be larger than $0^{\circ}$ ). Its corresponding GA command window, Figure 14, provides information of the number of generation required, convergence criteria, best fitness value and optimal wedge angles. Comparing Figures 12 and 14, both final points are quite close to each other, $(14.977,18.813)$ vs. $(15.039,18.779)$. It is due to the fact that the global maximum total pressure recovery (i.e. a global minimum of objective function) is located inside the feasible domain. It can also be seen from the surface plot of the objective function in Figure 15. It is an indication that this test case is a well defined physics-based model and serves well as a validation in this study. 


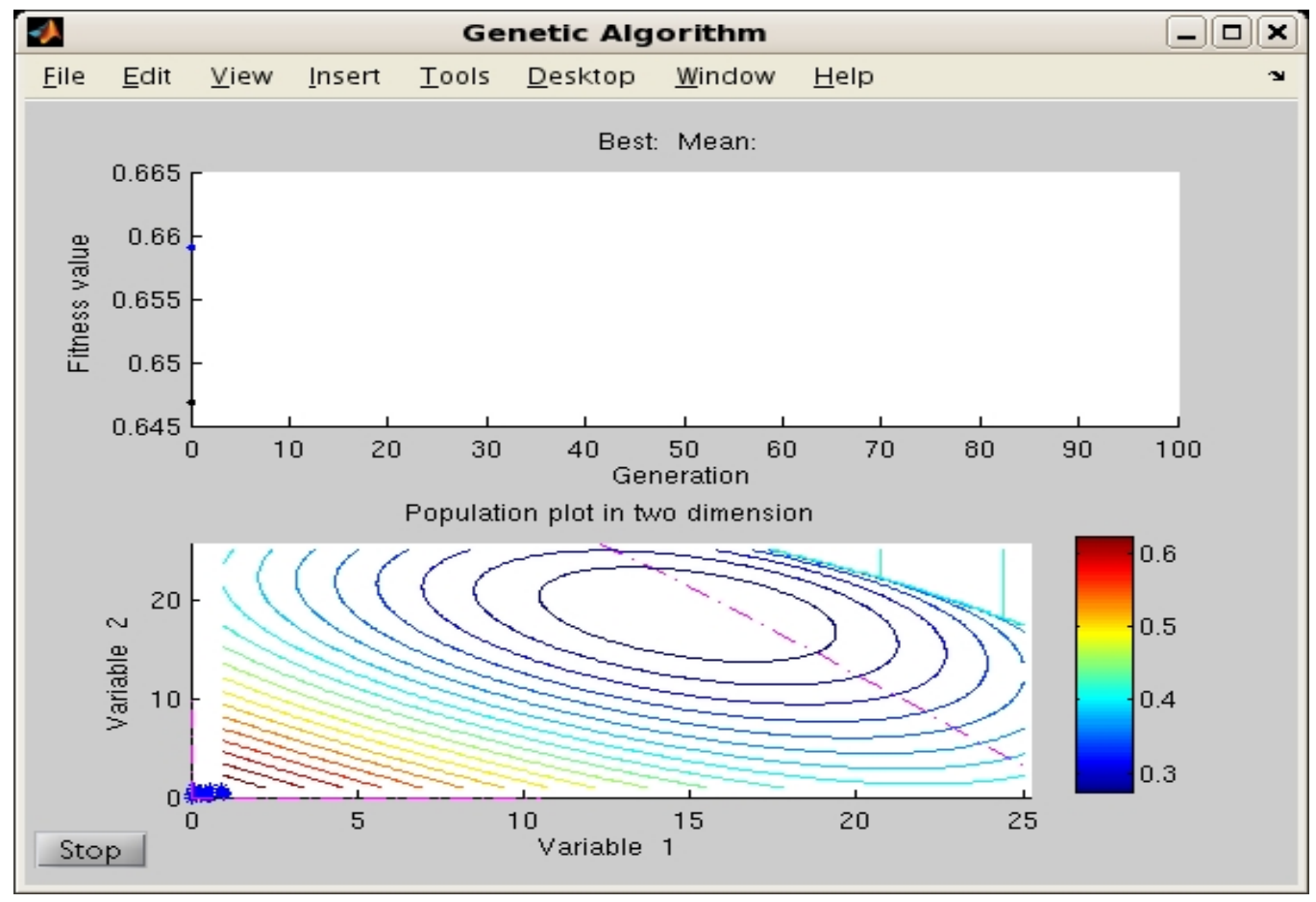

Figure 8: The upper plot shows the best (in black) and the mean (in blue) fitness value at the initial state, with * represent the initial distribution of population in the plot at the lower plot. The varible1 and variable2 are the first and second wedge angles respectively, with their lower bounds in magenta color. Also, the lower plot also displays the contours of objective function and dashed line is the nonlinear constraints used in this problem, $\tan \left(\delta_{1}\right)+\tan \left(\delta_{1}+\delta_{2}\right)$ $\leq 1$.

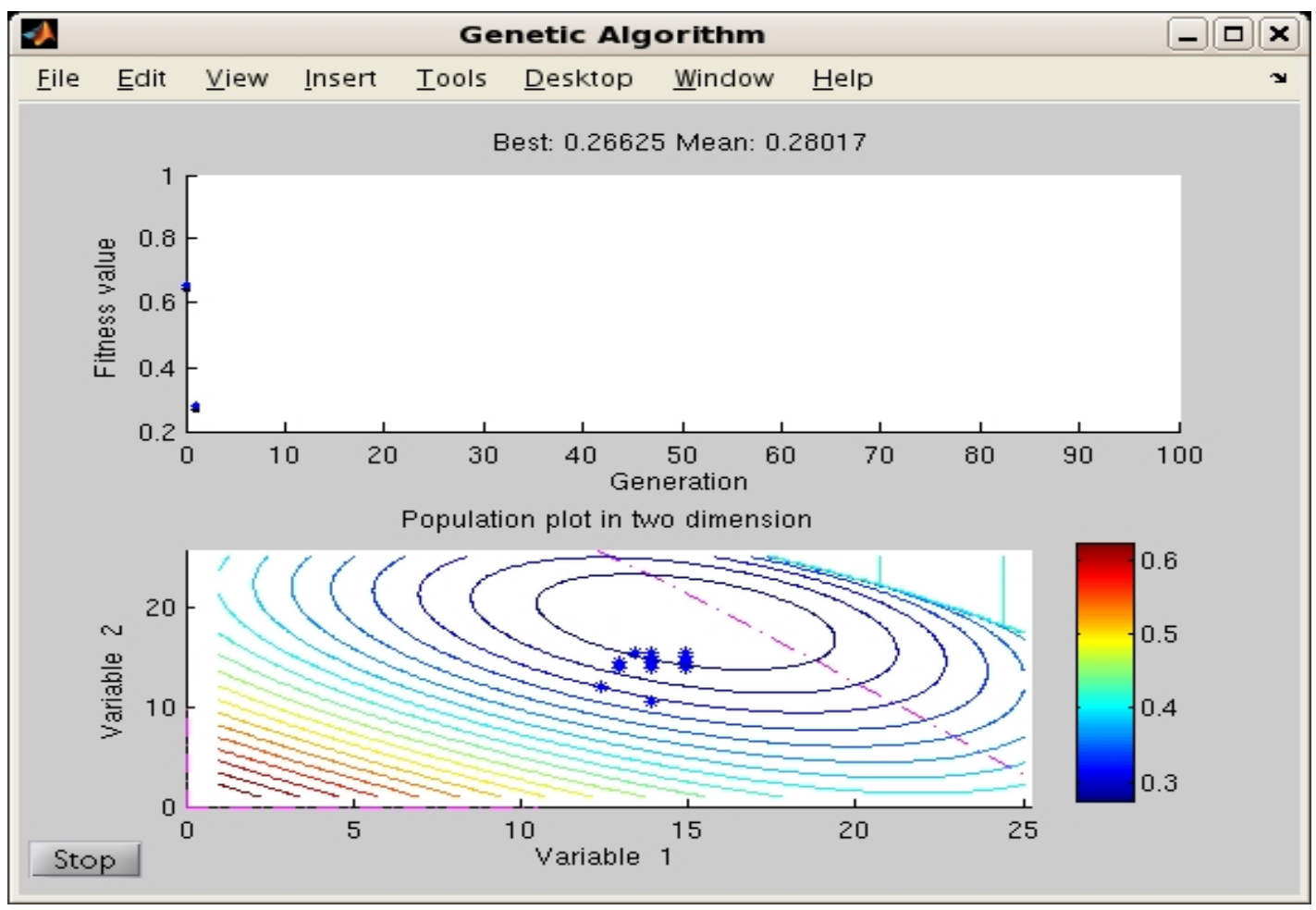

Figure 9: Continuing the evolution from the initial stage, the upper plot displays the best, mean fitness values in each generation. The lower plot displays the population distribution in the first generation. 


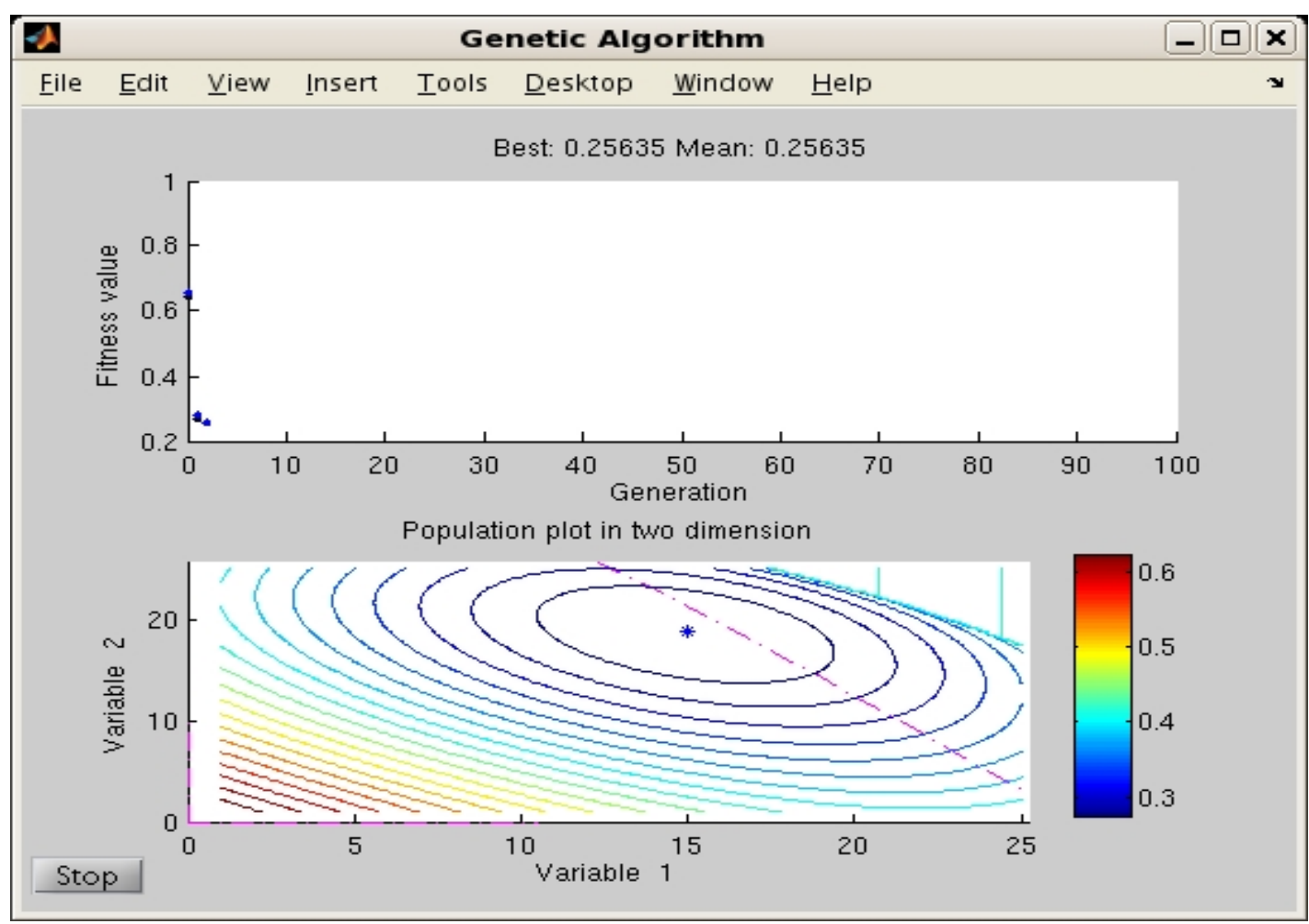

Figure 10: Continuing the evolution from the first generation, the upper plot displays the best, mean fitness values in each generation. The lower plot displays the population distribution in the second generation.

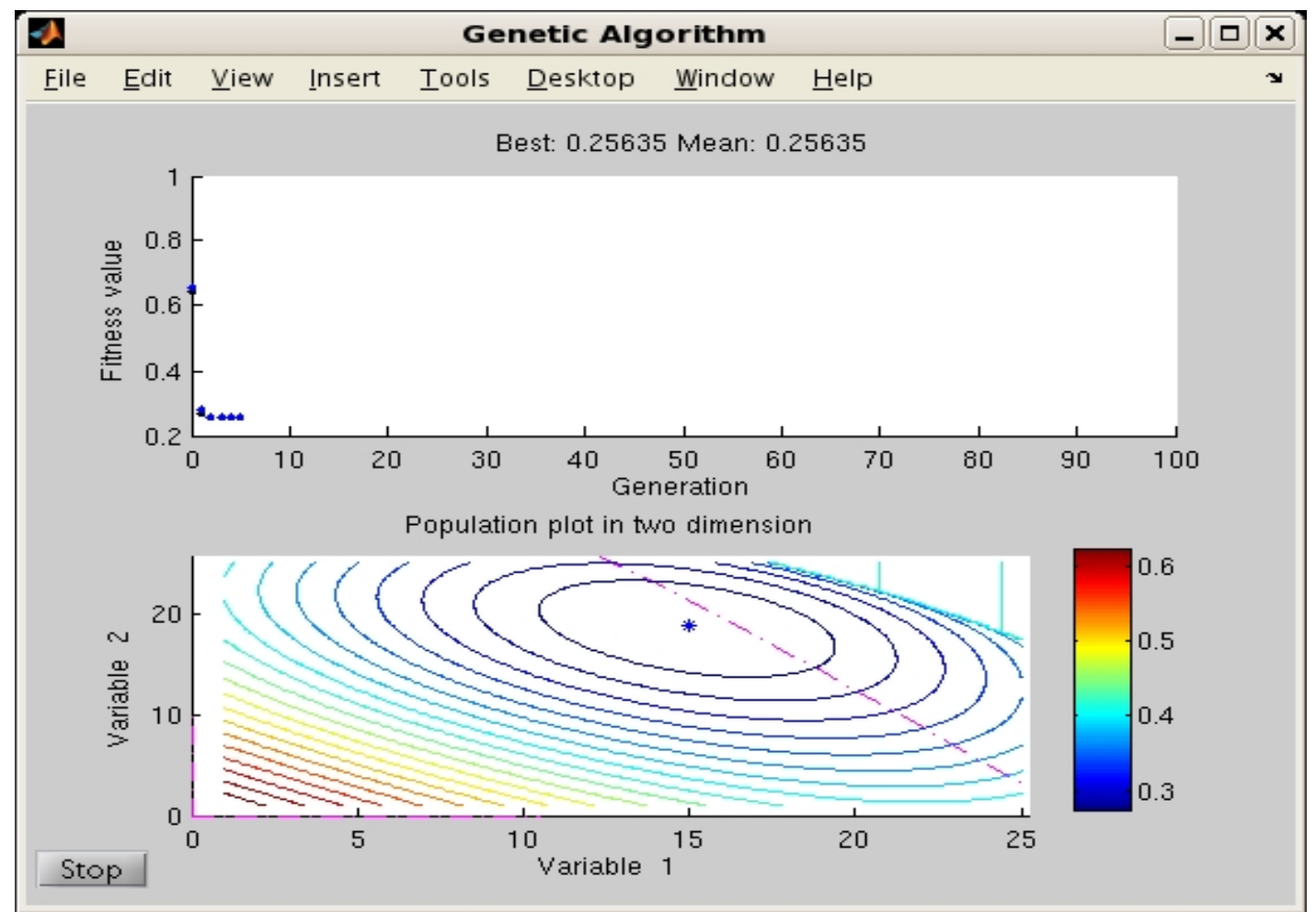

Figure 11: Continuing the evolution from previous generations, the upper plot displays the best, and mean fitness values in each generation. The lower plot displays the population distribution in the final converged stage. 


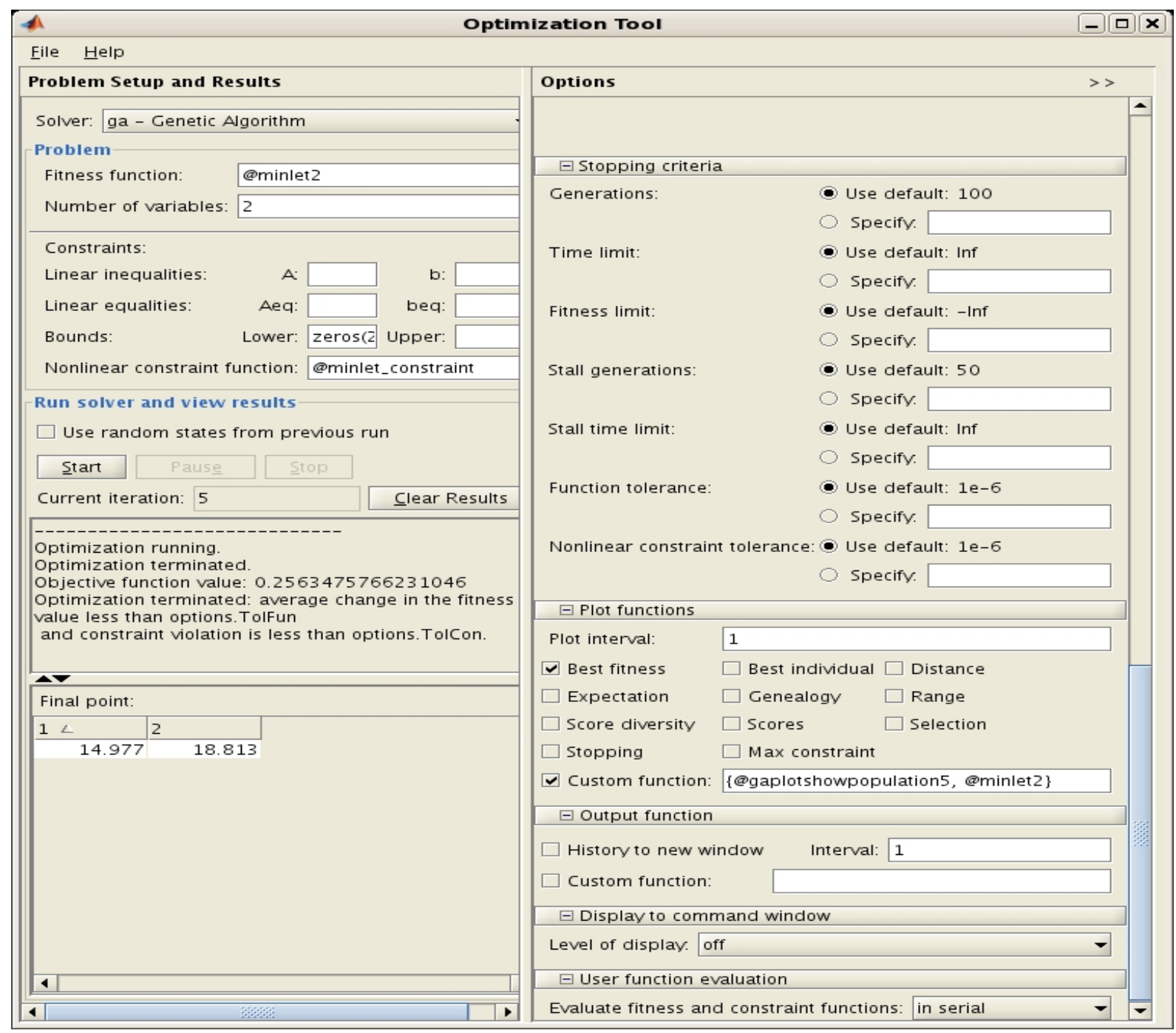

Figure 12: Genetic Algorithm command panel which allows users to input the objective function, number of design variables, and to set population size, mutation and crossover values, convergence criteria, etc. This figure shows the setup of Mach 3 test case.

More studies are made for different inflow conditions, ranging from inflow Mach number 2 to 5 . The optimum angle combination of the first and second wedge angles and its corresponding optimum pressure recovery are compared with those obtained by Oswatitsch. Figure 16 is a plot same as in $\operatorname{Secton}^{6}$ and shows how the pressure recovery varies with number of shock and free-stream Mach number. In the chosen double wedge geometry, $n=3$ in Oswatitsch notation, those GA results of optimum combinations at free-stream Mach number from 2 to 5 are indicated in black solid circles in Figure 16. The agreement is obvious. For Mach number form 1.5 to 4 , the optimized angle combinations produced by the genetic algorithm are plotted against those taken from Connors and Meyer ${ }^{7}$ in Figure 17. The agreement between two sets of optimum angle combinations, $\left(\delta_{1}, \delta_{2}\right)$, is excellent. In summary, Table 1 tabulates optimum performance and angle combinations for double-oblique-shock inlets at various $\mathrm{M}_{\infty}$.

\begin{tabular}{|l|l|l|l|l|l|l|l|l|}
\hline $\mathrm{M}_{\infty}$ & 1.5 & 2.0 & 2.5 & 3.0 & 3.5 & 4.0 & 4.5 & 5.0 \\
\hline$\left(\delta_{1}, \delta_{2}\right)$ & $\begin{array}{l}\left(5.55^{\circ}, 5.0\right. \\
\left.6^{\circ}\right)\end{array}$ & $\begin{array}{l}\left(10.41^{\circ}, 11.1\right. \\
\left.3^{\circ}\right)\end{array}$ & $\begin{array}{l}\left(13.36^{\circ}, 15 .\right. \\
\left.73^{\circ}\right)\end{array}$ & $\begin{array}{l}\left(14.97^{\circ}, 18 .\right. \\
\left.81^{\circ}\right)\end{array}$ & $\begin{array}{l}\left(15.81^{\circ}, 20 .\right. \\
\left.78^{\circ}\right)\end{array}$ & $\begin{array}{l}\left(16.14^{\circ}, 22\right. \\
\left..1^{\circ}\right)\end{array}$ & $\begin{array}{l}\left(16.21^{\circ}, 22 .\right. \\
\left.92^{\circ}\right)\end{array}$ & $\begin{array}{l}\left(16.07^{\circ}, 23 .\right. \\
\left.48^{\circ}\right)\end{array}$ \\
\hline $\begin{array}{l}\text { Total } \\
\text { pressure } \\
\text { recovery }\end{array}$ & 0.995 & 0.957 & 0.868 & 0.744 & 0.610 & 0.487 & 0.383 & 0.3 \\
\hline
\end{tabular}

Table 1: Optimum of total pressure recovery and its corresponding angle combination for double-oblique-shock supersonic inlets by using the genetic algorithm, where $\mathbf{M}_{\infty}$ is the free stream Mach number. 


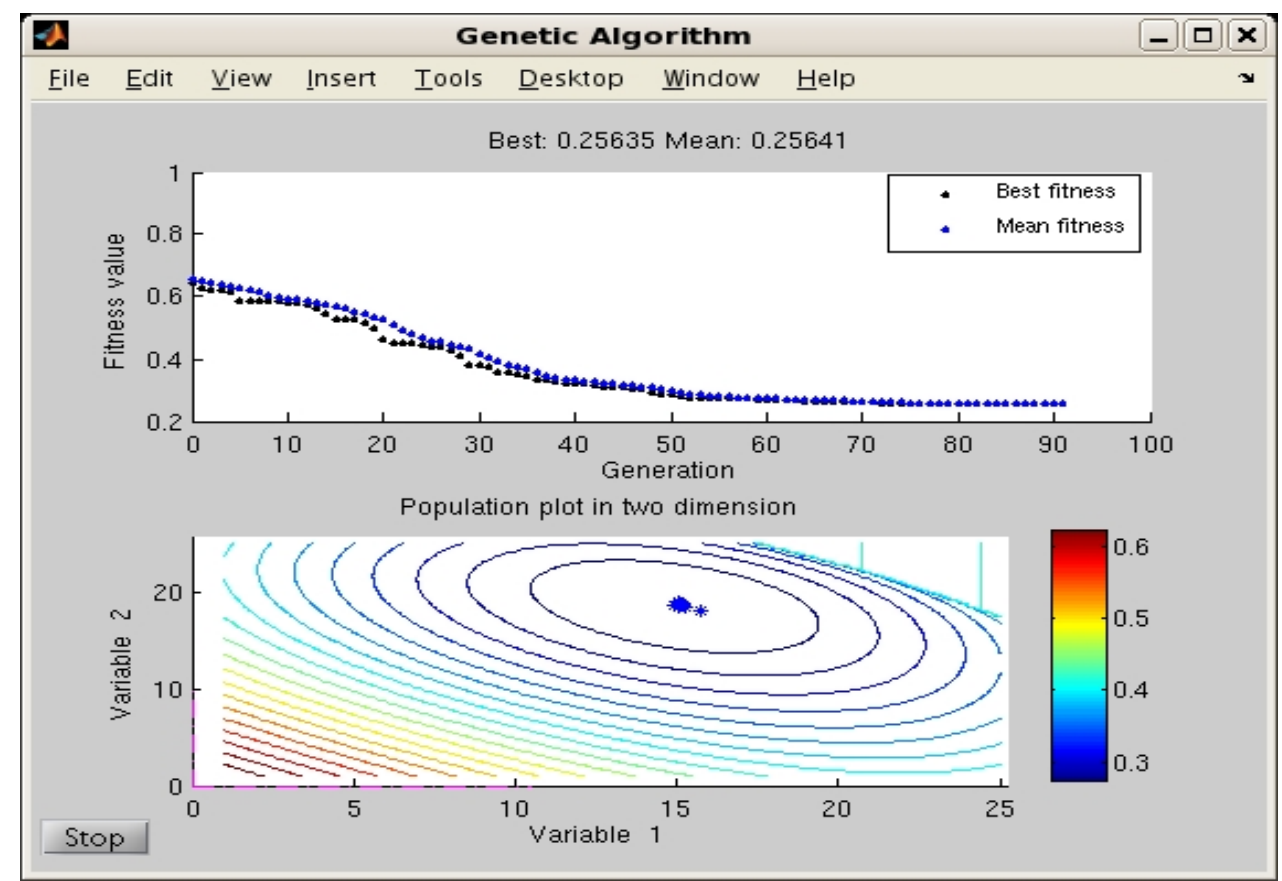

Figure 13: At final stage of a no constraint GA run, the upper plot displays the objective function value of mean and best fitness values in each generation. The lower plot displays the final population distribution, bounds and contours of objective function.

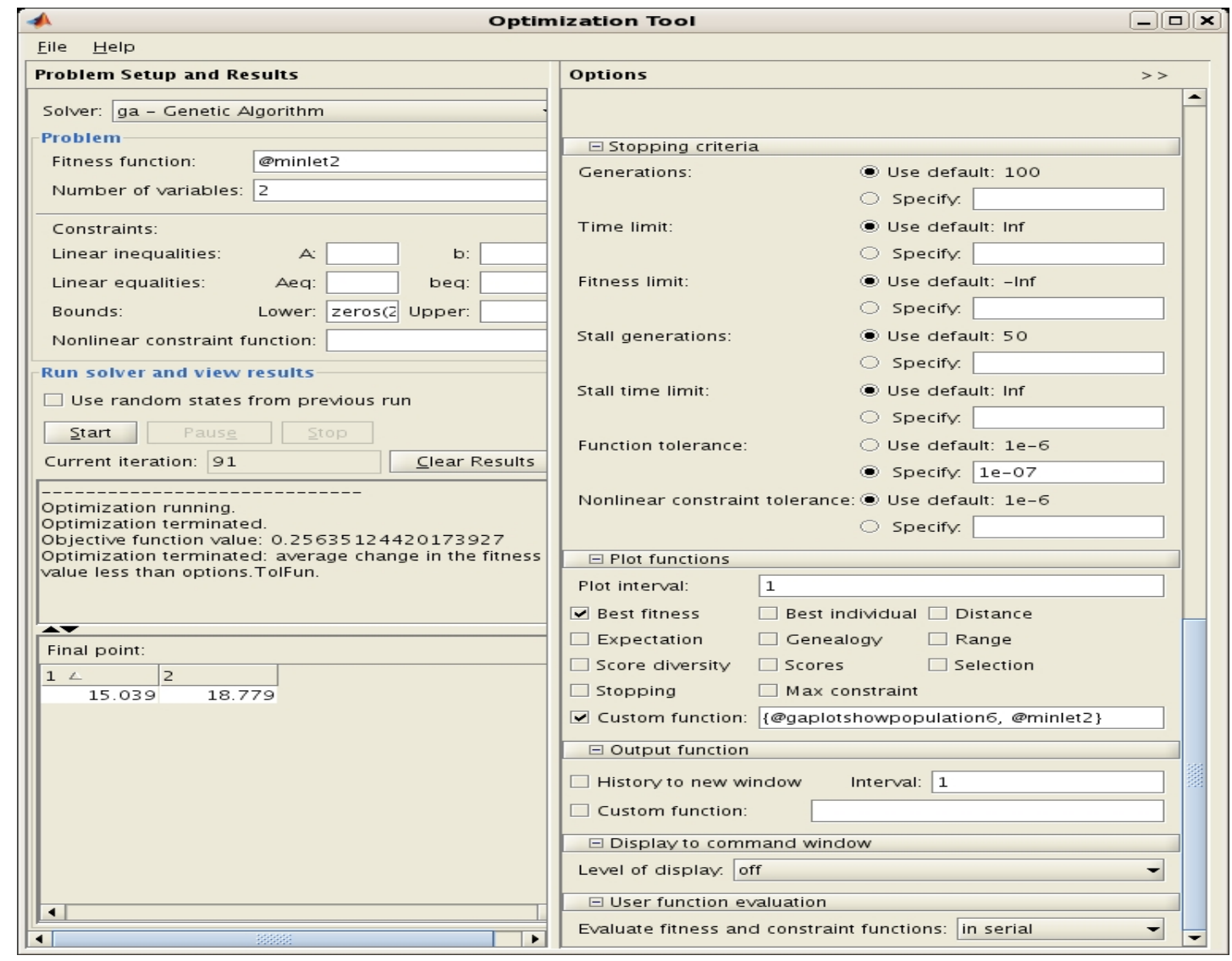

Figure 14: Genetic Algorithm command panel which allows users to input the objective function, number of design variables, and to set population size, mutation and crossover values, convergence criteria, etc. This figure shows the setup of Mach 3 test case without imposing constraint. 


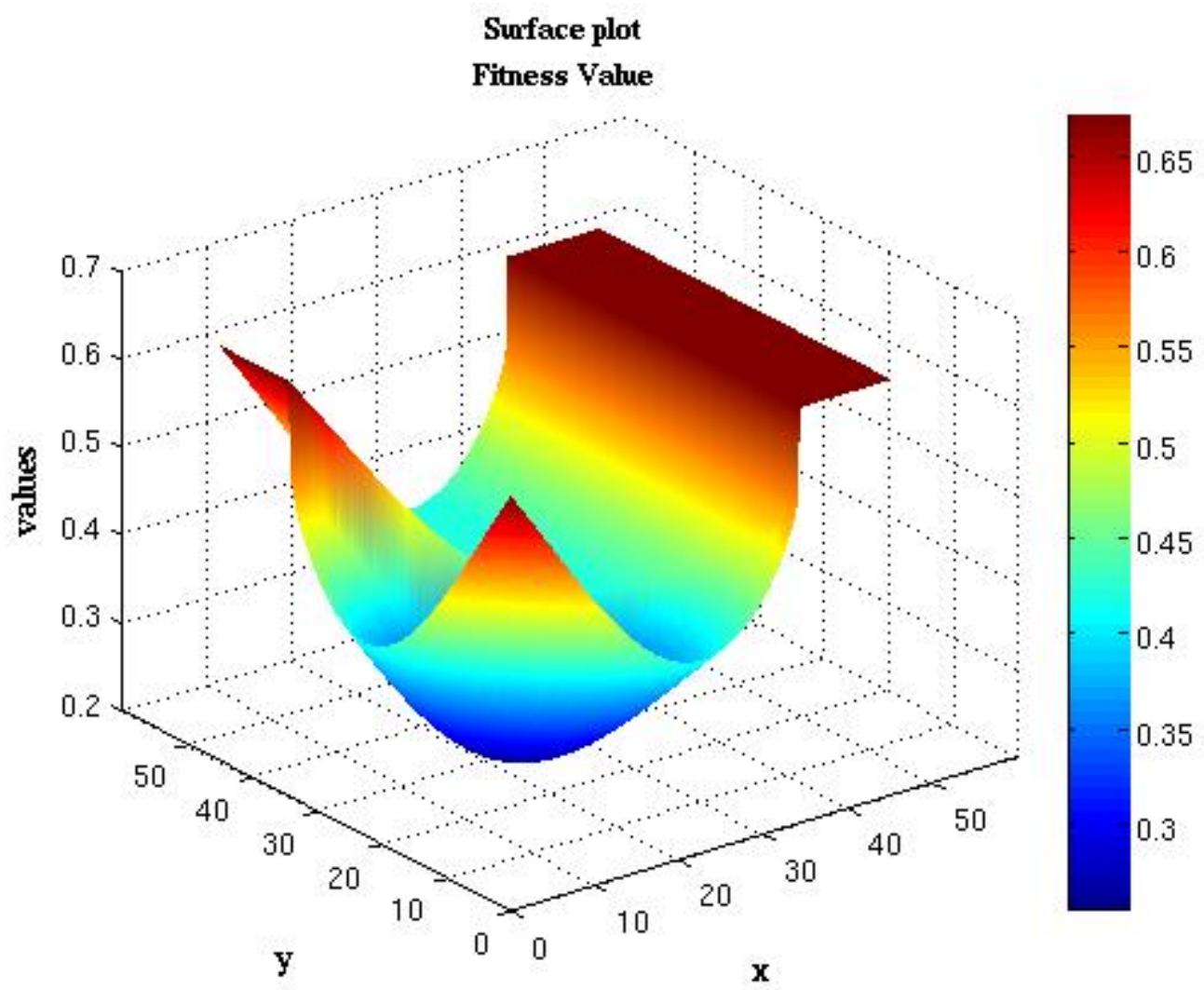

Figure 15: Surface plot of Oswatitsch shock theory at Mach 3, with two oblique shocks and one normal shock.

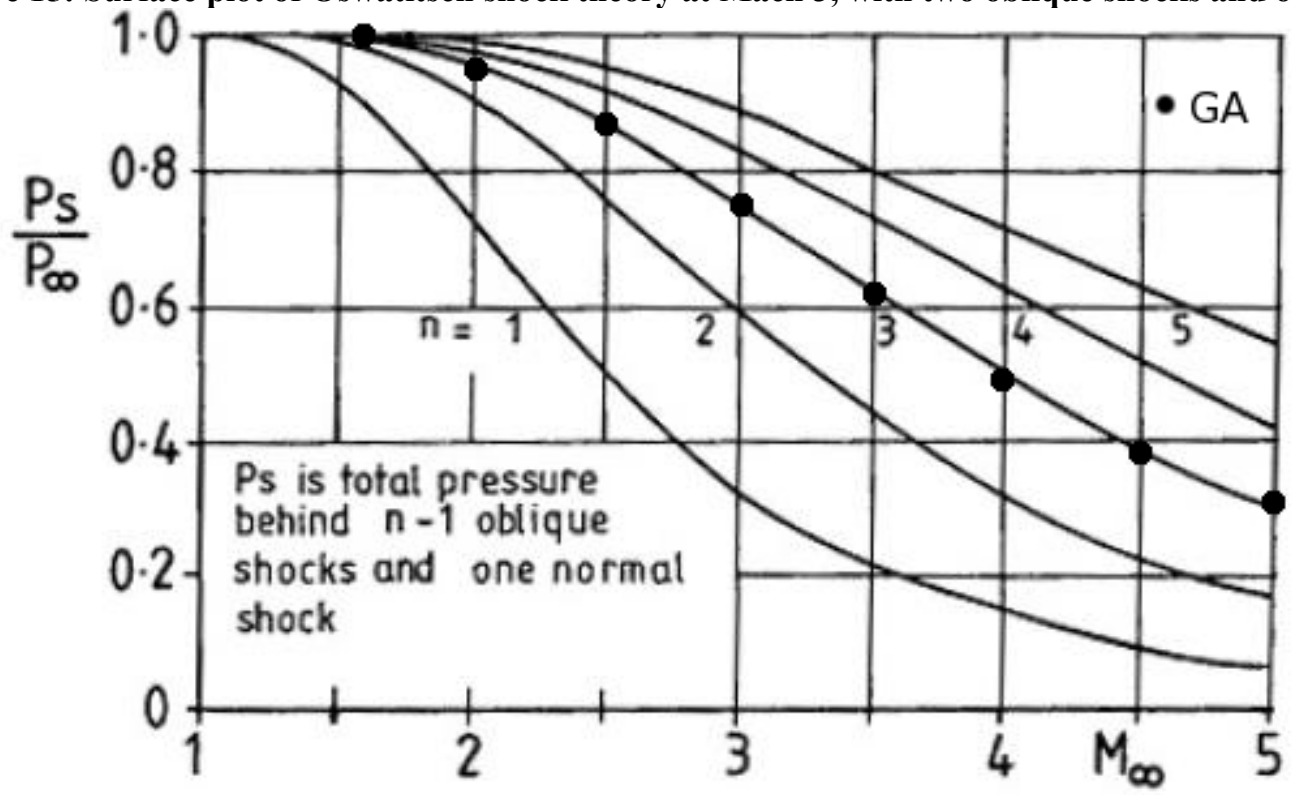

Figure 16: Shock pressure recovery for optimum arrangements of two-dimensional shocks; the genetic algorithm's results are indicated by solid circles, with Oswatitsch results in solid lines which are taken from Ref. 6.

This test case has successfully demonstrated that the chosen optimizer is able to deliver accurate optimum results against known theoretical solution. The other test case is a viscous case. In stead of using NACA 1135 equations, the viscous calculation of a similar type of problem as Oswatitsch's is carried out using PNS solver and meshes generated by Gridgen in automatic mode. The goal is to look for the best combination of wedge deflection angles in a double wedge inlet, as same as the other test case. The genetic algorithm returns an expected solution that flat plate has the least total pressure loss. Similarly, the best and mean history, population distribution, and GA command panel are presented in Figure 18 and 19. Figure 19 presents the optimum combination of $(0.051,0.882)$. Physics governs the design again. 


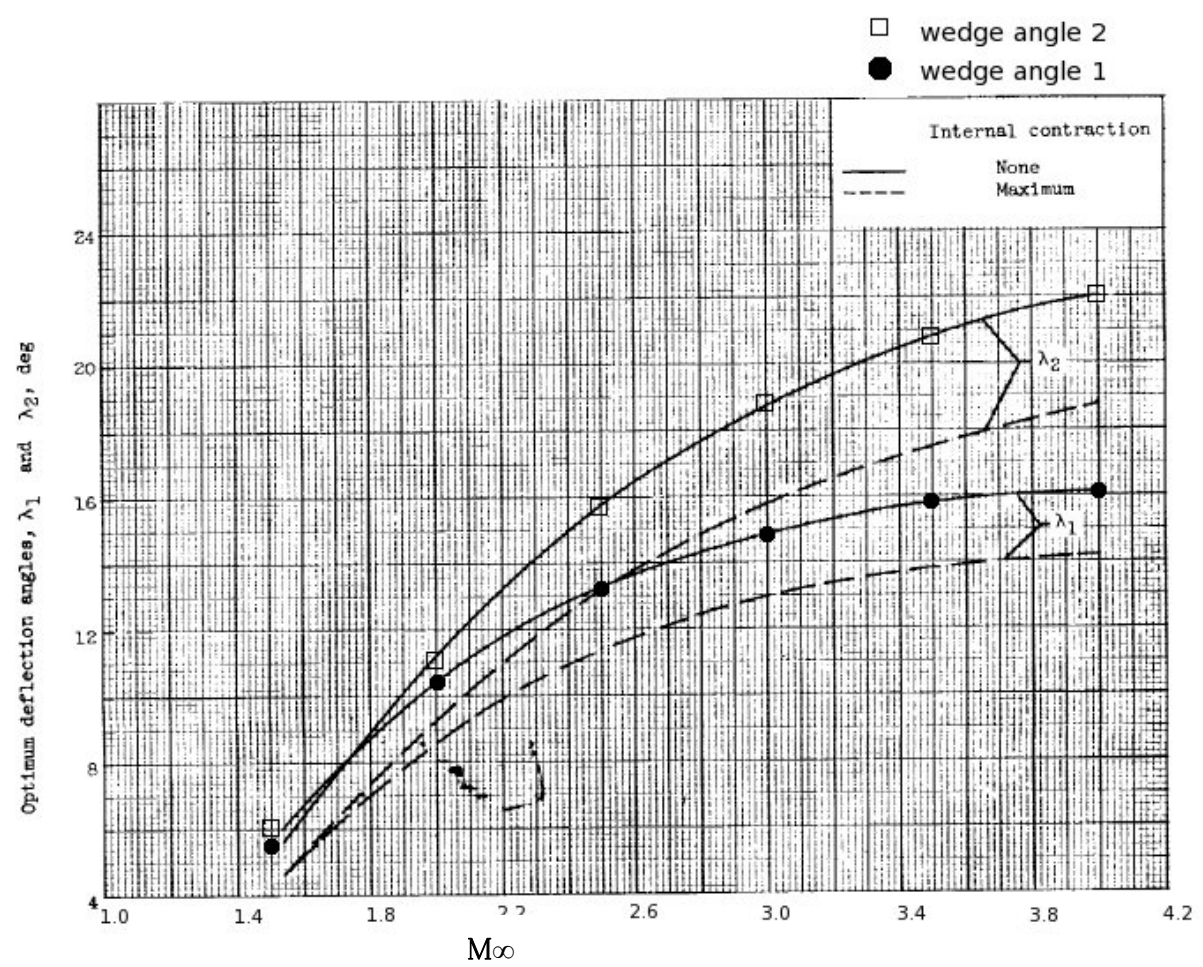

Figure 17: The angle combinations for double-oblique-shock supersonic inlets in optimum performance; symbols of - and $\square$ indicate the GA results of wedge angle 1 and 2 respectively from free stream Mach number 1.5 to 4 .

Best: 0.23231 Mean: 0.23231
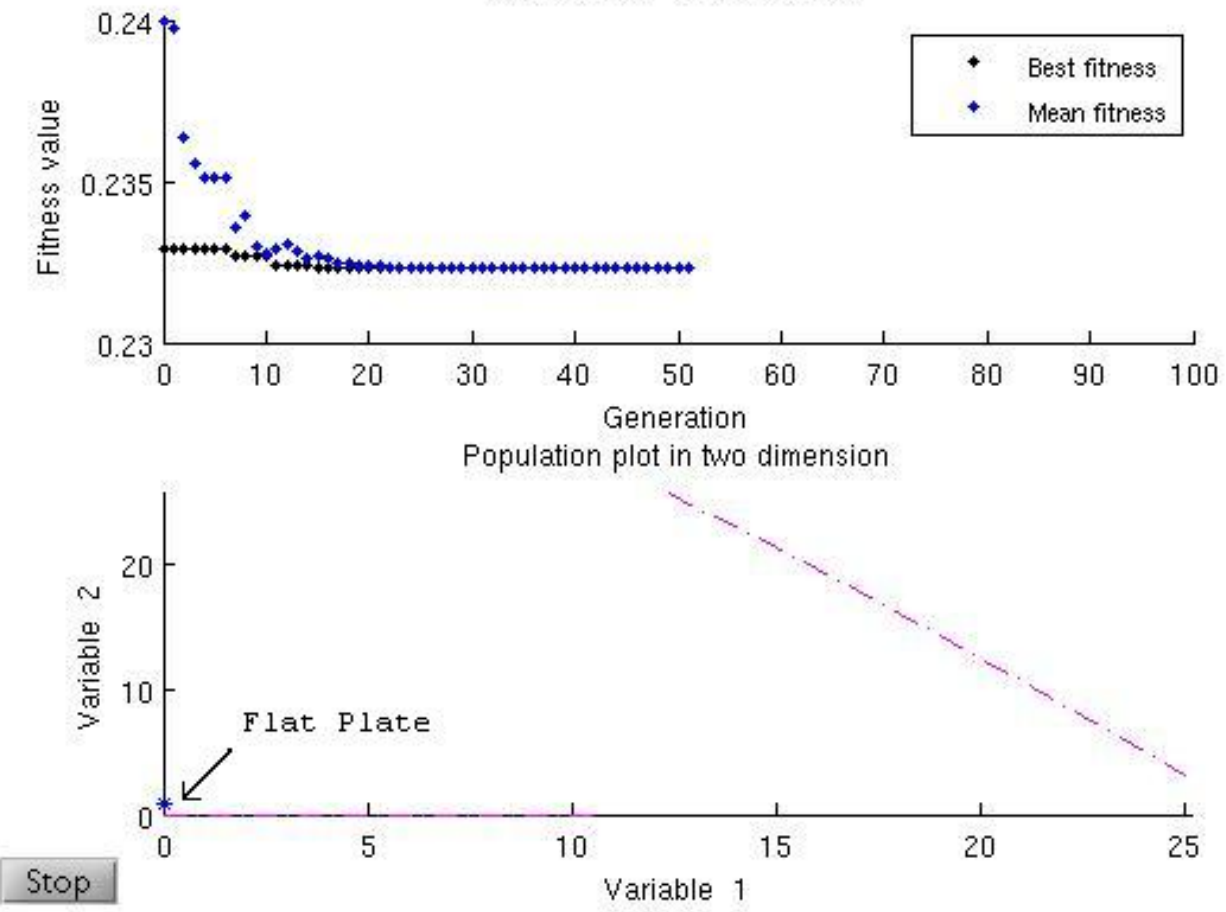

Figure 18: The upper plot displays the mean and best fitness values in the final generation. The lower plot displays the population distribution at the current generation, showing what GA returns optimum is close to a flat plate. 


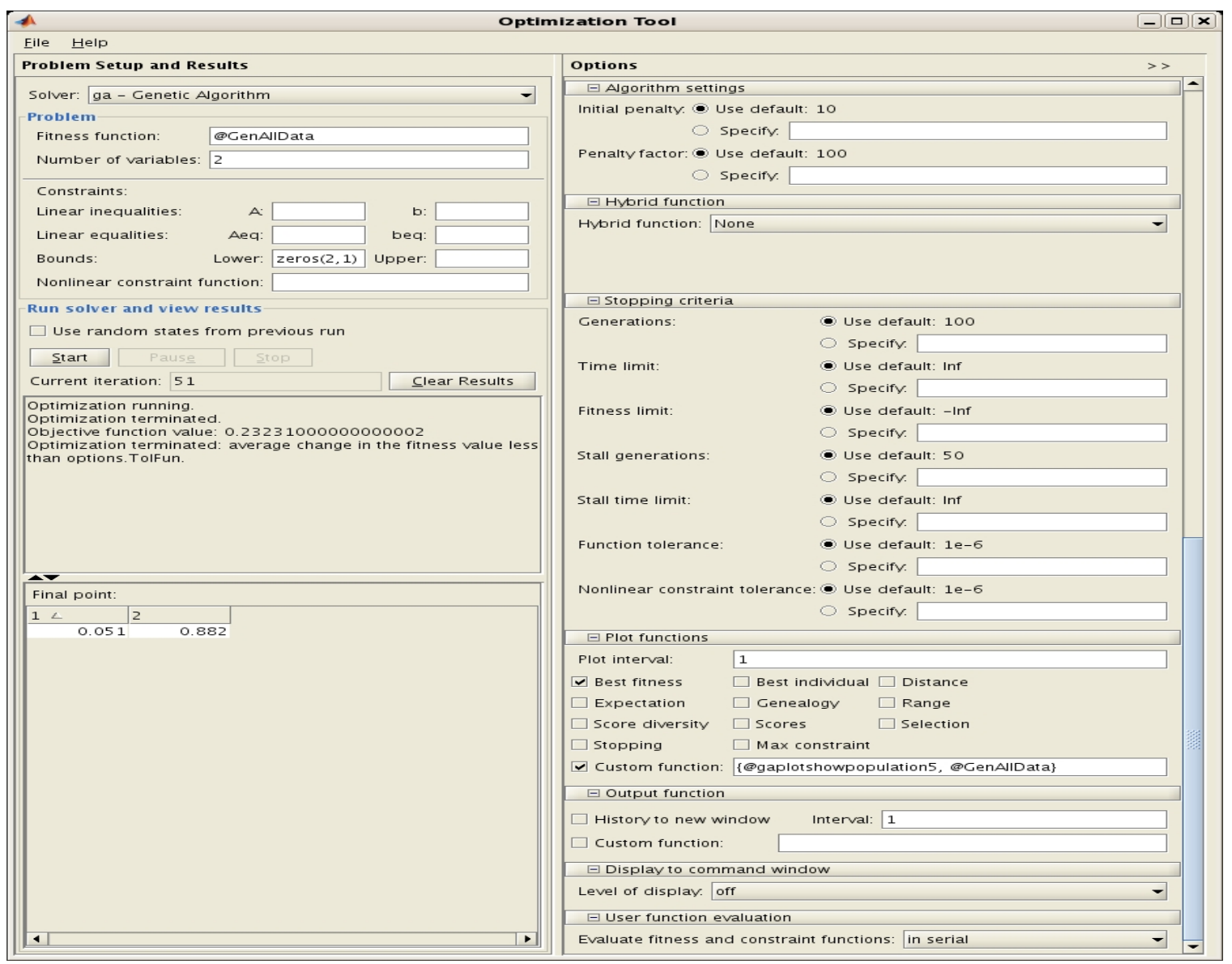

Figure 19: The GUI of MATLAB optimization tool displays information on the solver used, defined problem, number of design variables, constraints, options selected, and final point of the design variables leading to the best fitness value.

\section{Inlet design showcase}

In the following cases, a baseline 2D inlet has two ramps, with eight-degree deflection angle of each. The first ramp is one and a half feet long, followed with an equal length second ramp, and the lower surface turns straight afterwards. A moveable cowl is placed 1 foot above and can be at various distances from the ramp leading edge, the same type of geometry as in other studies. ${ }^{8}$ The free stream Mach number is 3.0 and Reynolds number at 4,830,000 per foot. A Mcdonald-Camarata mixing length turbulence $\operatorname{model}^{9}$ is used and the walls of the inlet are assumed to be adiabatic. The 80x800 meshes are packed near wall. With the number of design variables increasing gradually from 2 to 4 , more complexity is added to inlet geometric design.

\section{Approach 1: Two design variables}

\section{Case 1: two wedge angles, no cowl}

As stated before, two-wedge ramp is constructed and the genetic algorithm returns a flat plate like geometry. After adding a constraint with $\delta_{1}+\delta_{2} \geq 15^{\circ}$, the genetic algorithm returns the combination of two angles, $\left(6.851^{\circ}, 8.148^{\circ}\right)$ which falls right on the constraint line. Figure 20, the Mach number contours clearly indicate the growth of boundary layer along the lower ramp surface. This case matches the known facts: a less deflected ramp results in less total pressure loss, and the equal or close to equal shock strength, $\mathrm{M}_{1} \operatorname{Sin} \theta_{1} \approx \mathrm{M}_{2} \operatorname{Sin} \theta_{2}$ as shown in Figure 7 , gives better recovery.

\section{Case2: one wedge angle and moveable cowl}

To improve upon the performance of the baseline inlet geometry, geometric modifications are carried out by GA. In this case, the first wedge angle $\left(\delta_{1}\right)$ is fixed at $8^{\circ}$ while the second angle $\left(\delta_{2}\right)$ becomes a design variable, variable 1 . The other design variable is the cowl leading edge location $\left(\mathrm{X}_{\text {cowl }}\right)$ which is the variable 2 . In this GA run, $\delta_{2}$ has a lower bound of $0^{\circ}$ and is bounded by an inequality of $20^{\circ}$, which is arbitrary set based on the known fact of inlet aerodynamics. 
Therefore, the bounds of the deflection angle of the second ramp is

$$
0^{\circ} \leq \delta_{2} \geq 20^{\circ} \text {. }
$$

The cowl location is set between 1.5 and 2.5 feet, which is imposed as inequality constraints on variable 2 . Constraints and bounds on both variables are plotted in Figure 22 in magenta dotted line.

The optimum design is the elimination of the second wedge, i.e. $\delta_{2}=0^{\circ}$, based on what the genetic algorithm returns. In Figure 21, the both static pressure and Mach number contours show the final second wedge angle is zero, also shown in Figure 22 of the population distribution at final GA generation. This is a violation of the known fact in aerodynamics that for a given Mach number, the more oblique shocks, the higher pressure recovery. As repeatedly stated, inlet design is a trade-off between factors. Among those factors, oblique shocks ahead of throat area and normal shock behind the throat area are two contradicting factors. The weaker oblique shocks are in the front, the stronger normal shock is behind. What the genetic algorithm minimized is based on the total pressure loss from solver. The PNS solver used in the system can not account for the normal shock effect. To provide the genetic algorithm a more realistic evaluation of the total pressure recovery, the theoretical NACA 1135 normal shock calculation is added to compensate the PNS solver's inability in handling normal shock. Therefore, a normal shock is presumed at the exit face of the computational domain and its strength is determined by the averaged Mach number at the exit. As a result, the normal shock effect is included in the recalculation of this case and the following cases.
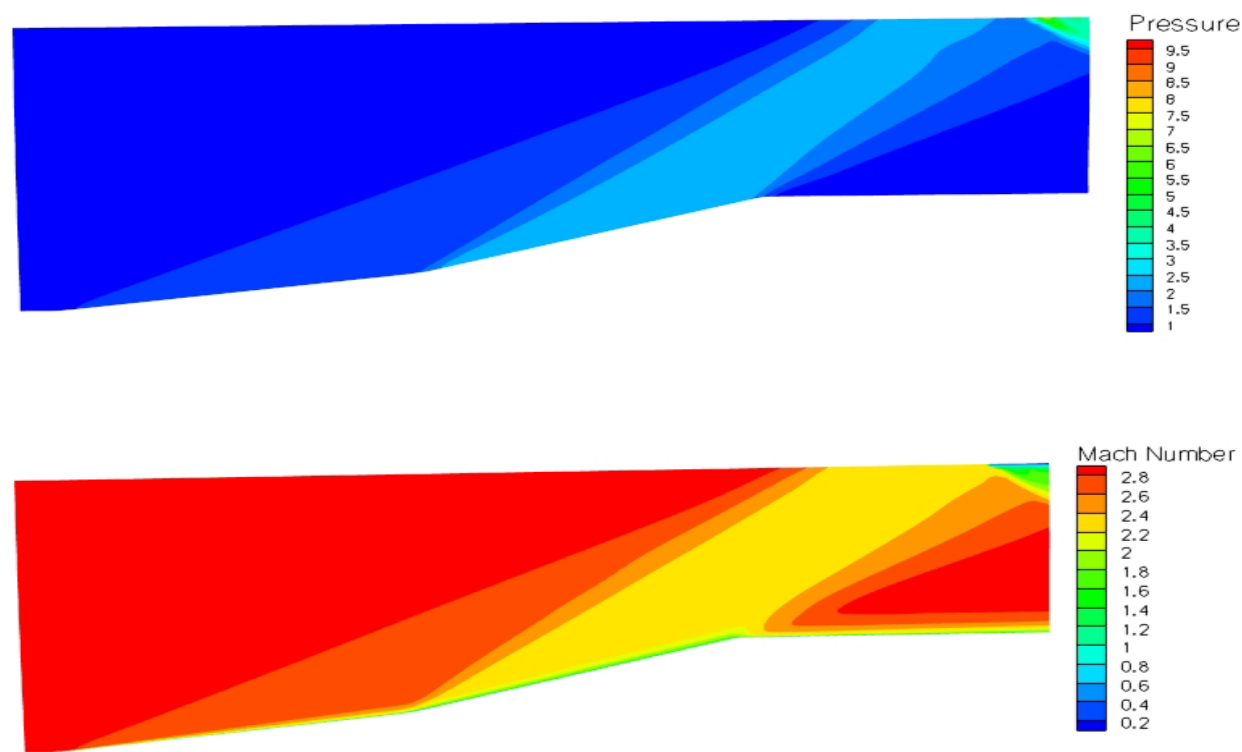

Figure 20: The static pressure and Mach number contours of the inlet in Case 1.
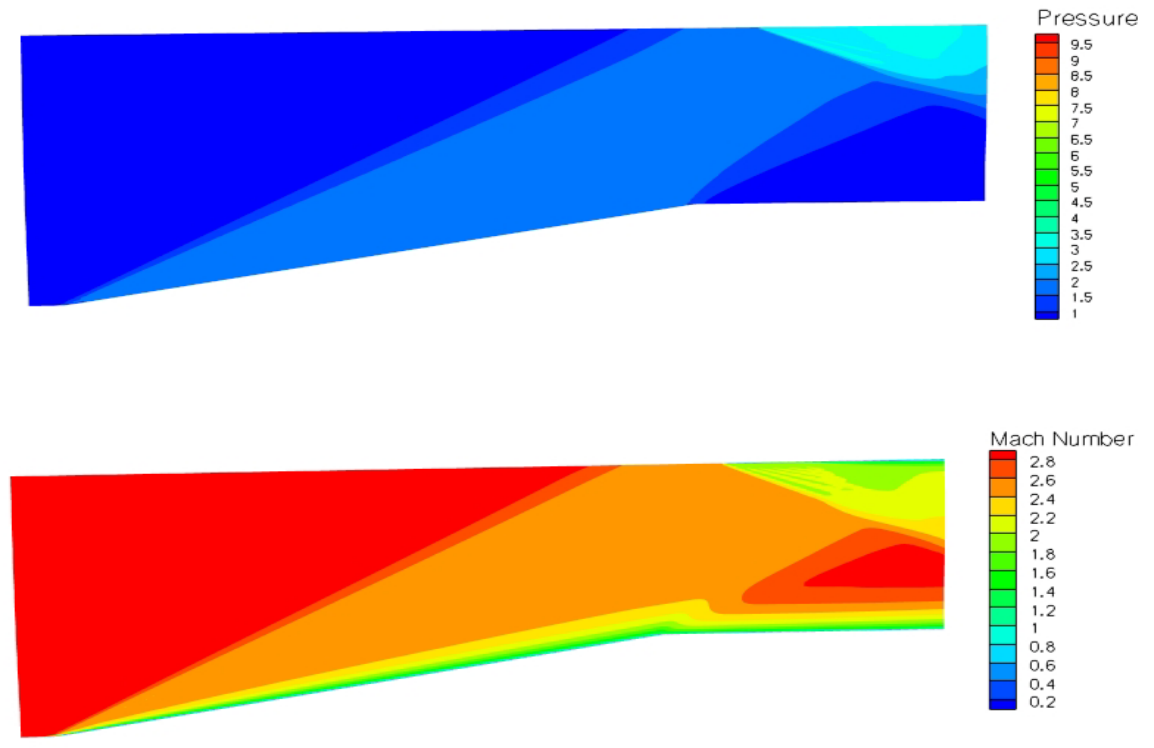

Figure 21: Static pressure and Mach number contours of the inlet in Case 2, no normal shock considered. 


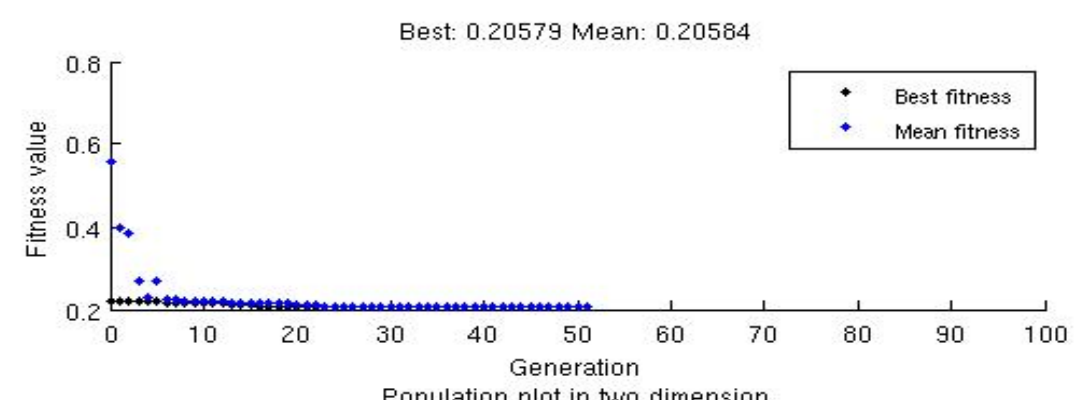

Population plot in two dimension

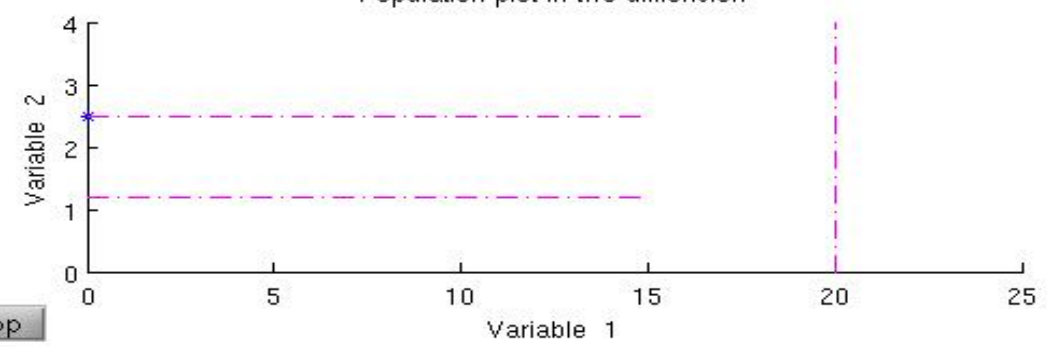

Figure 22: The upper plot displays the best and mean fitness values in each generation. The lower plot display the population distribution indicated by $*,\left(0^{\circ}, 2.5 \mathrm{ft}\right)$ at the final generation. Variable 1 is the deflection angle, $\delta_{2}$, and Variable 2 is the distance of the cowl lip from the leading edge of the inlet, $X_{\text {cowl }}$.
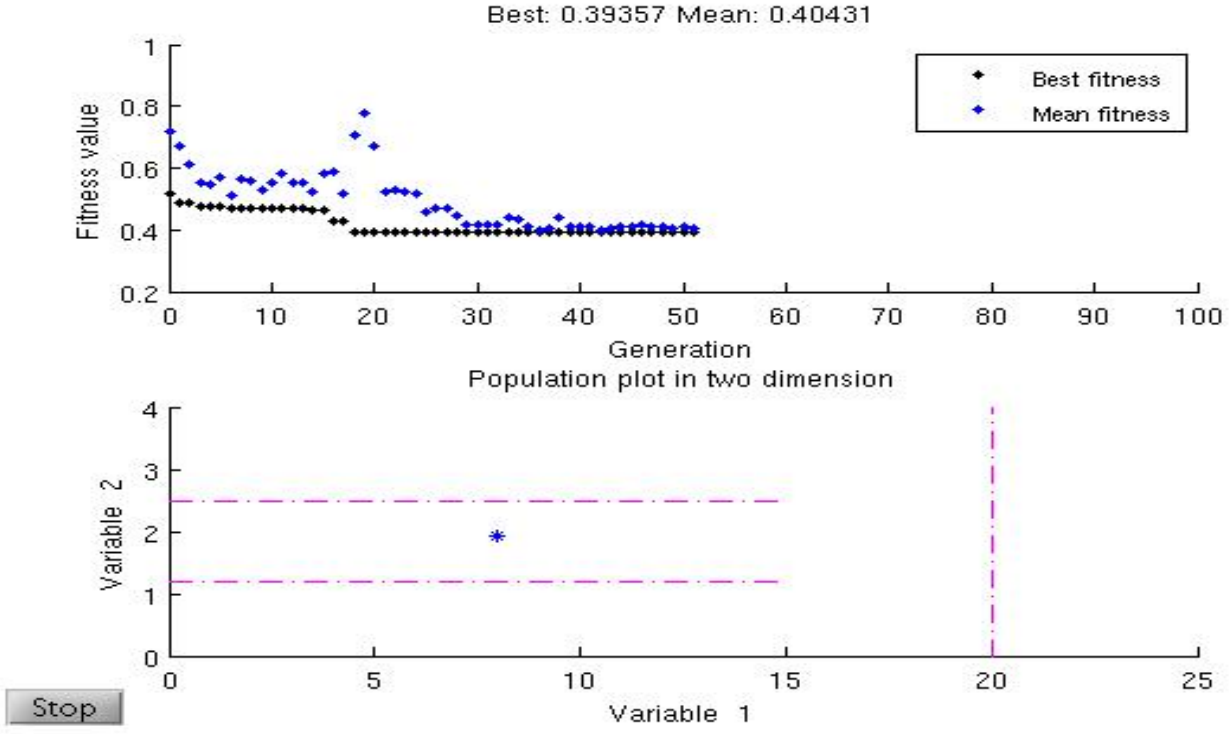

Figure 23: The upper plot displays the best and mean fitness values in each generation. The lower plot display the population distribution indicated by ${ }^{*},\left(7.985^{\circ}, 1.928 \mathrm{ft}\right)$ at the final generation. Variable 1 is the deflection angle, $\delta_{2}$ and Variable 2 is the distance of the cowl lip from the leading edge of the inlet, $X_{\text {cowl }}$.

After adding the consideration of normal shock effect, the newly created optimum geometry is shown in Figure 24 by the static pressure and Mach number contours. In Figure 23 of the GA plots, the variable 1 in the lower plot, i.e. $\delta_{2}$ at the final generation, is no longer laying on its lower bound of $0^{\circ}$. Even though the total pressure recovery at the exit face before the imposed normal shock is worse than the one in Figure 21, with value of 0.7145 vs. 0.738. But, the total pressure recovery after the terminal shock is much better, with 0.606 vs. 0.447 . 

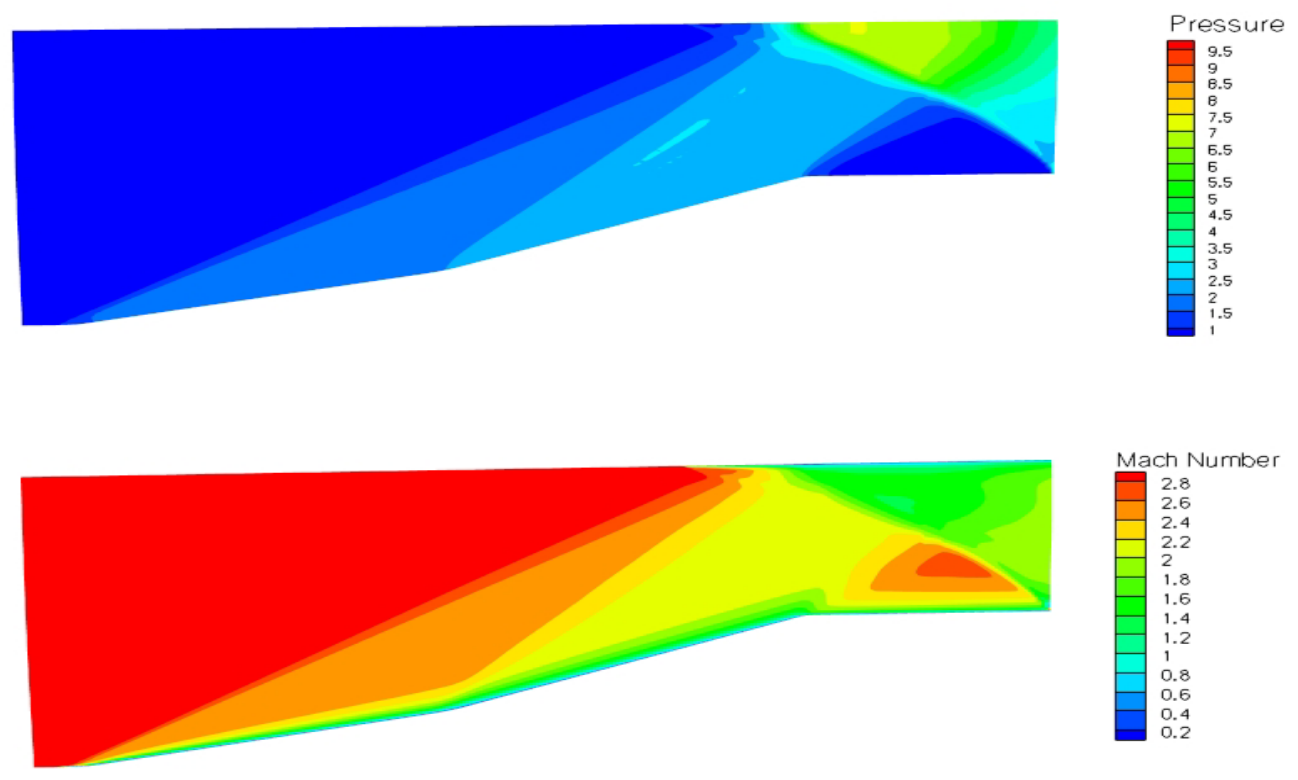

Figure 24: The static pressure and Mach number contours of the inlet geometry which is the result of the trade off among oblique and the normal shock. The normal shock is appended at the end of the inlet.

Case 3: double ramps, fixed cowl and an isentropic portion near throat

Considering the supersonic section of an inlet, the critical Mach number is at the throat, which determines the strength of the downstream normal shock and the static pressure at the compressor face further down. A nearly isentropic geometry is desirable for its minimal total pressure loss. Based on previous studies and Oswatitsch theory, a design of a number of weak oblique shock waves and a weak normal shock just behind the throat to decelerate the flow to subsonic conditions can produced least total pressure losses. Also, it is known that isentropic compression waves are preferred over the oblique shock in supersonic portion of the inlet. With that in mind, a task in finding a geometric shape of the ramp at lower surface in which the gradual compression of the flow can be nearly isentropic. The baseline geometry as described before is used. But, the second ramp now is divided into two halves. The cowl leading edge is fixed at $X_{\text {cowl }}=2.0$. The shape of the second half in ramp 2 is replaced with a cubic polynomial function,

$$
\mathbf{y}=\mathbf{y}_{2}+\mathbf{a} *\left(\mathbf{x}_{3}-\mathbf{x}_{2}\right)+\mathbf{b}^{*}\left(\mathbf{x}_{3}-\mathbf{x}_{2}\right)^{2}+\mathbf{c} *\left(\mathbf{x}_{3}-\mathbf{x}_{2}\right)^{3}
$$

where $\mathrm{a}$ is the slope of the third ramp. Intuitively, the third ramp is given a slope continuingly from the second ramp and leaves the coefficients of the second and the third order terms as design variables, i.e. $a=\tan \left(\delta_{2}\right)$. Therefore, the final geometry has become a two- wedge ramp with an additional curve section in front of the shoulder where the lower surface turning flat toward the exit. Note, the final point from GA should be values of $b$ and $c$, i.e. $(b, c)$.

The genetic algorithm returns a ramp shaped for a compression zone near the throat area is created. The static pressure and Mach number contours are given in the Figure 25. Mach number contours in Figure 25 clearly indicate the growth of boundary layer along the lower ramp surface and the upper cowl surface. The shock waves from ramps and cowl are shown by the change in color contour. An abrupt change of color indicates shock location. An improvement from Case 2, the flow is compressed through a series of compression waves after the second ramp, and then expanded at the shoulder as shown in the pressure contours in Figure 25. There are several complex compressible flow phenomena in this case, including intersection of ramp shocks, and intersection of the reflected ramp shocks with the compression waves from the last part of ramp and the expansion from the shoulder of the lower surface near the exit face. The plot of the best and mean fitness values over the entire generations are displayed in Figure 26.

\section{Approach 2: Three design variables}

Case 4: triple ramps with fixed cowl lip location

Exploring further the geometry of Case 2, the coefficient a is assigned to be another design variable. In other words, coefficients $\mathrm{a}, \mathrm{b}$, and $\mathrm{c}$ in the equation above are optimized by the genetic algorithm. The cowl lip is fixed at two feet from the leading edge of the lower ramp surface. Note, although flow compression has to be gradual which implies the length of this section can not be overly constrained, the length of this section is fixed at a constant, one half of a foot. The optimizer is expected to create a curved ramp turning flow as isentropic as possible. It turns out that a third ramp is created, again 
considering maximizing total pressure recovery. A similar concaved curve in the third ramp generates expansion and compression waves before the flow turning into the shoulder at the lower right corner. There are three oblique shocks, compression waves and expansion fans incurred in this configuration. Flow is slightly expanded at the beginning of the third ramp before hitting the reflected shock from the cowl, which weakened the reflected shock a bit. Then the flow is compressed through a series of compression waves to reach the shoulder area. The resulting static pressure and Mach number contours are shown in Figure 27. Again, GA returns a geometry which qualitatively makes sense.
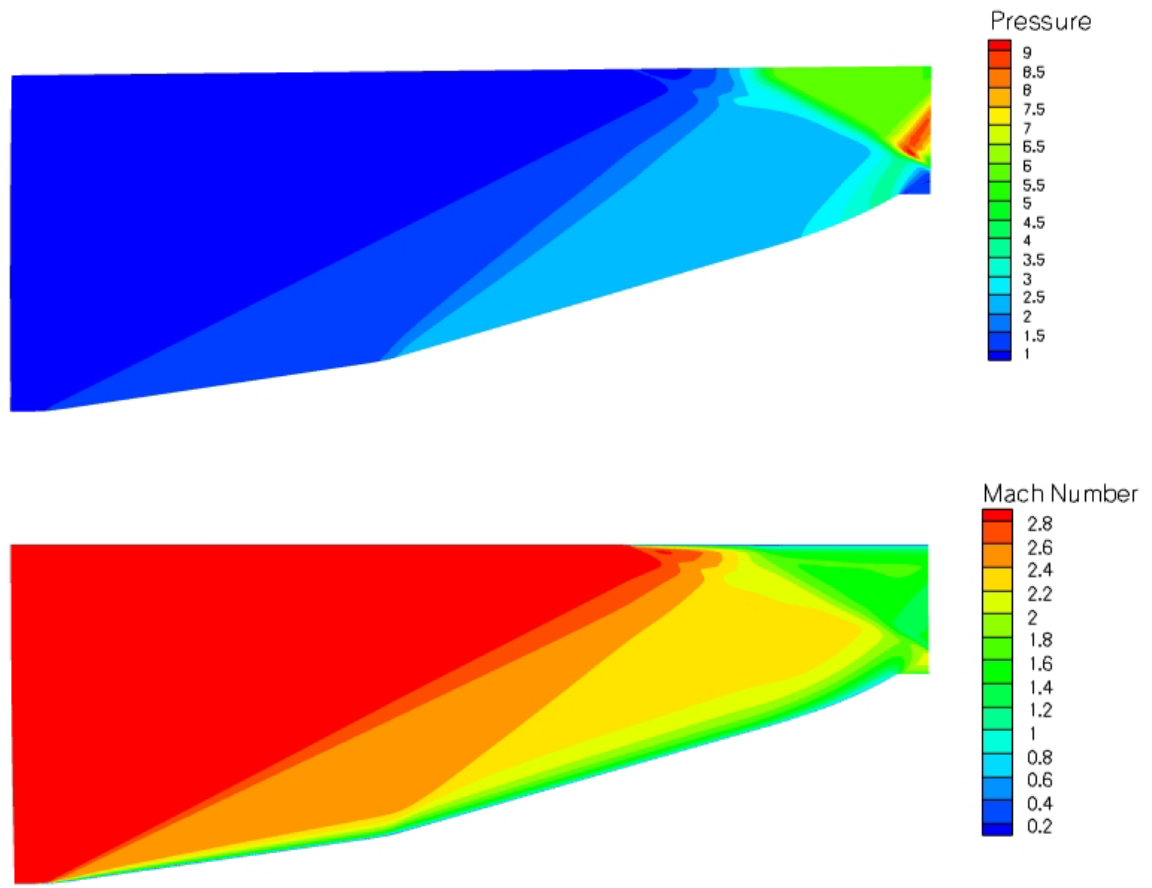

Figure 25: The static pressure and Mach number contours of the inlet in case 3.

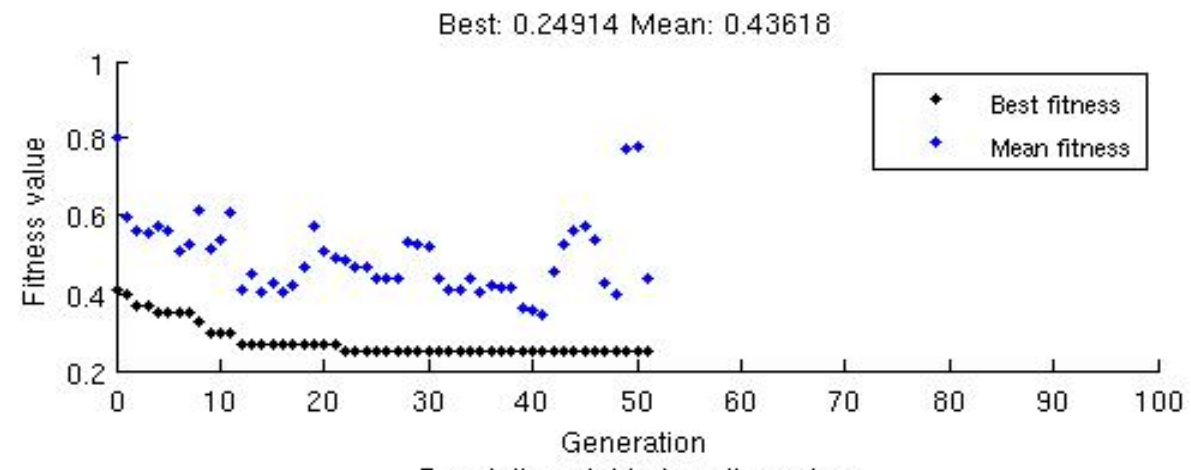

Population plot in two dimension

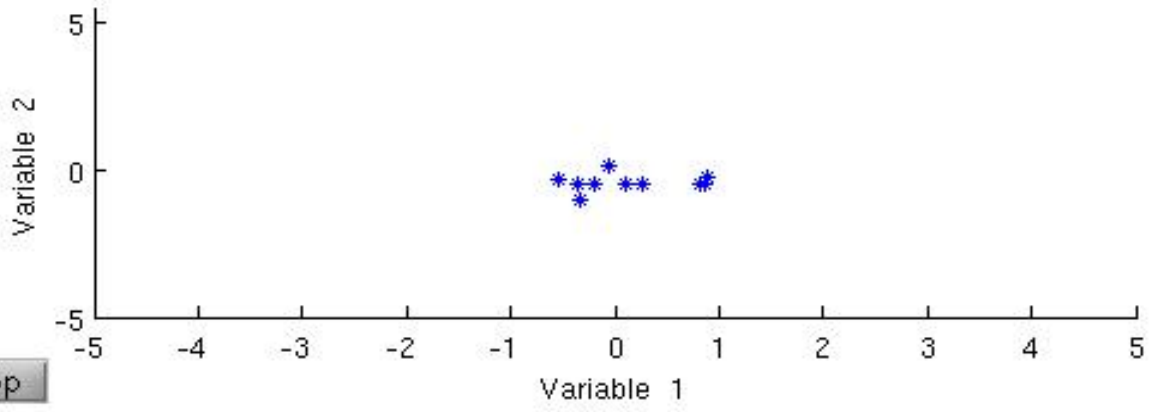

Figure 26: The upper plot displays the best and mean fitness values in each generation and the lower is the final state of the population distribution in Case 3 . Here, each individual is indicated by *. 

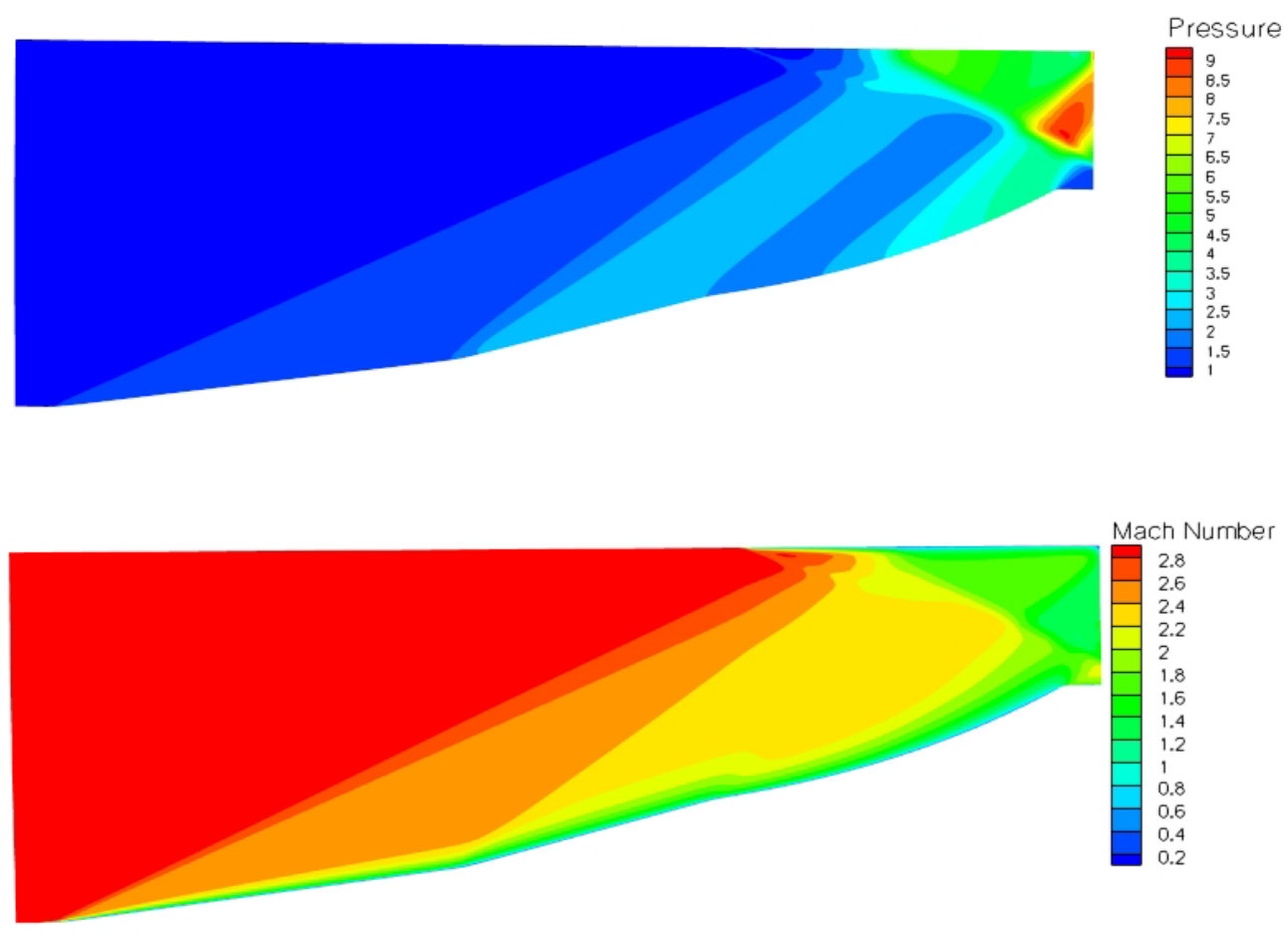

Figure 27: Static pressure and Mach number contours of the inlet in Case 4.

\section{Approach 3: Four design variables}

Case 5: triple ramps with moveable cowl lip

Adding complexity to the case 4, the cowl of the inlet is set to be movable. The geometric location of cowl leading edge, $\mathrm{X}_{\text {cowl }}$, is the fourth design variable. Considering the increasing number of design variables, individuals used in each generation are increased from default value of twenty to thirty. The resulting static pressure and Mach number contours are displayed in Figure 28. The final cowl location is shown in where the Mach contours indicate the growth of boundary layer at the upper surface. It looks like the first oblique shock falls behind the cowl lip. Then, the second oblique shock from the second ramp falls further inside the inlet top wall. In this configuration, these two oblique then are reflected from the cowl lip after hitting the top wall. At the lower surface, the shape of this triple-ramp geometry changes very gradually and flow decelerates and is compressed slowly till the flow encounters the reflected shock. The third weak oblique shock intersects the reflected shock and both shocks transmit to reach the opposite side. Then, the flow approaches the exit face after crossing expansion fans which are emitted from the shoulder. This expansion of flow apparently has weakened the reflected shock from the top. Thus, the flow remains supersonic, but meanwhile the Mach number is kept as low as possible before it reaches the exit. The flow filed is clean and simple.

Interestingly, a competing geometry which is only slightly less efficient in terms of fitness value (total pressure recovery) is shown in Figure 29. Two oblique shocks from the first and second ramps fall farther inside the top wall. In this geometry, the third ramp begins with concave shape, then is inflected into a convex curve. Corresponding to this shape, expansion fans appear first and slightly weaken the reflected shock from the cowl, then around the inflection point the resulting compression waves intersect the same reflected shock from the top surface and transmit to reach the opposite side, as shown in the Mach contours. Near the exit face, there was another set of expansion fans emitted from the shoulder of the lower ramp and the reflected shock is further weakened before it reaches the ramp. Mach number contours show a small area (blue color) of flow separation along the upper surface near the exit.

Also, another interesting geometry is evolved from GA's reproduction process. The geometry is defined by its static pressure and Mach number contours in Figure 30. Different from two previously described configurations, those two oblique shocks from the first and second ramps have coalesced near the cowl lip. They act more like external compressions, and then are reflected from the cowl lip after hitting it. At lower surface, a triple-ramp geometry is formed and flow is compressed first at the convex shaped third ramp before it is expanded along the turn toward shoulder. It appears there are small pockets of low speed flow after shocks crossing, but later re-energized by the expansions. Similarly, 
expansion waves weaken the reflected shock from the top.
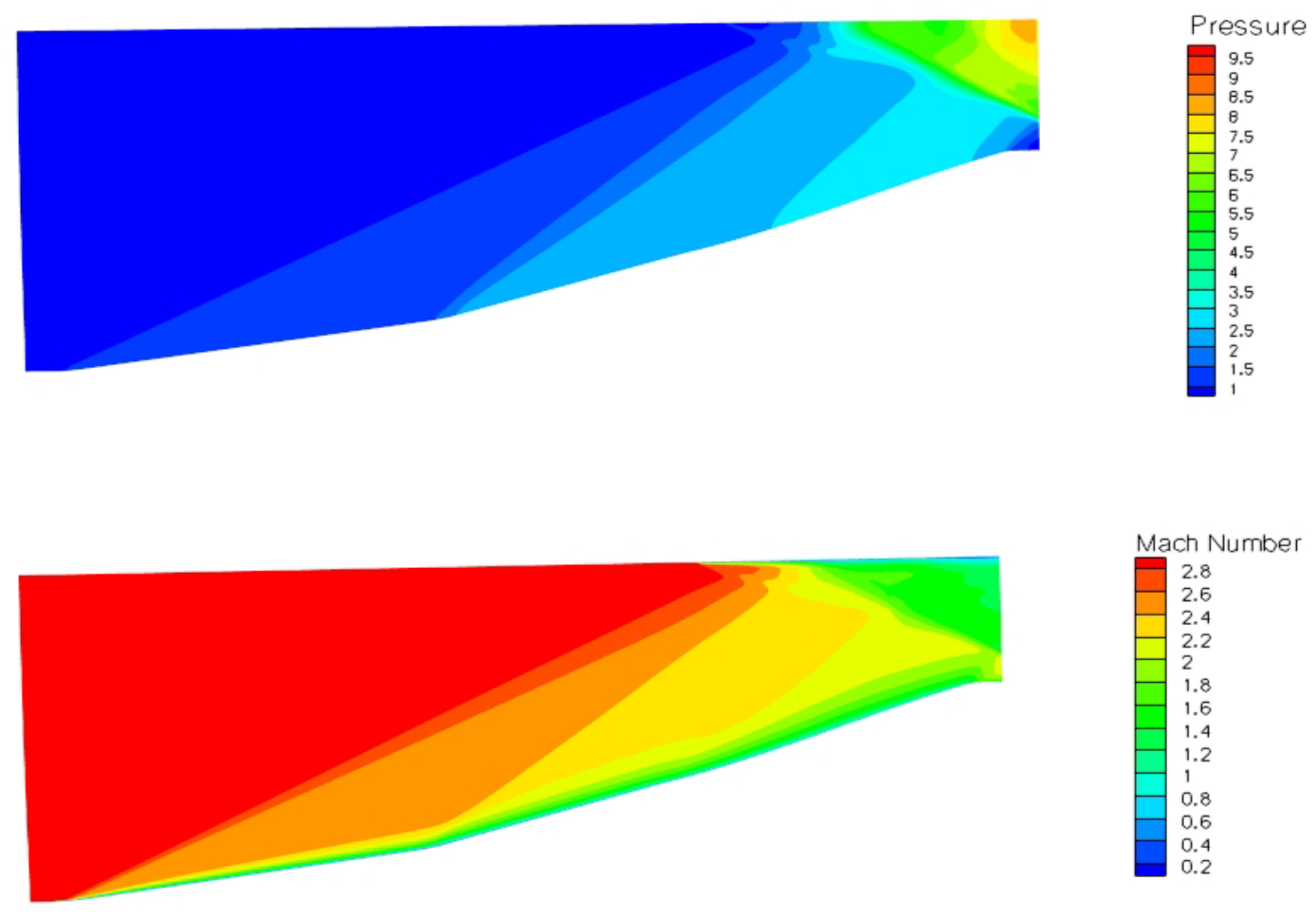

Figure 28: Static pressure and Mach number contours of the optimized inlet in Case 4.
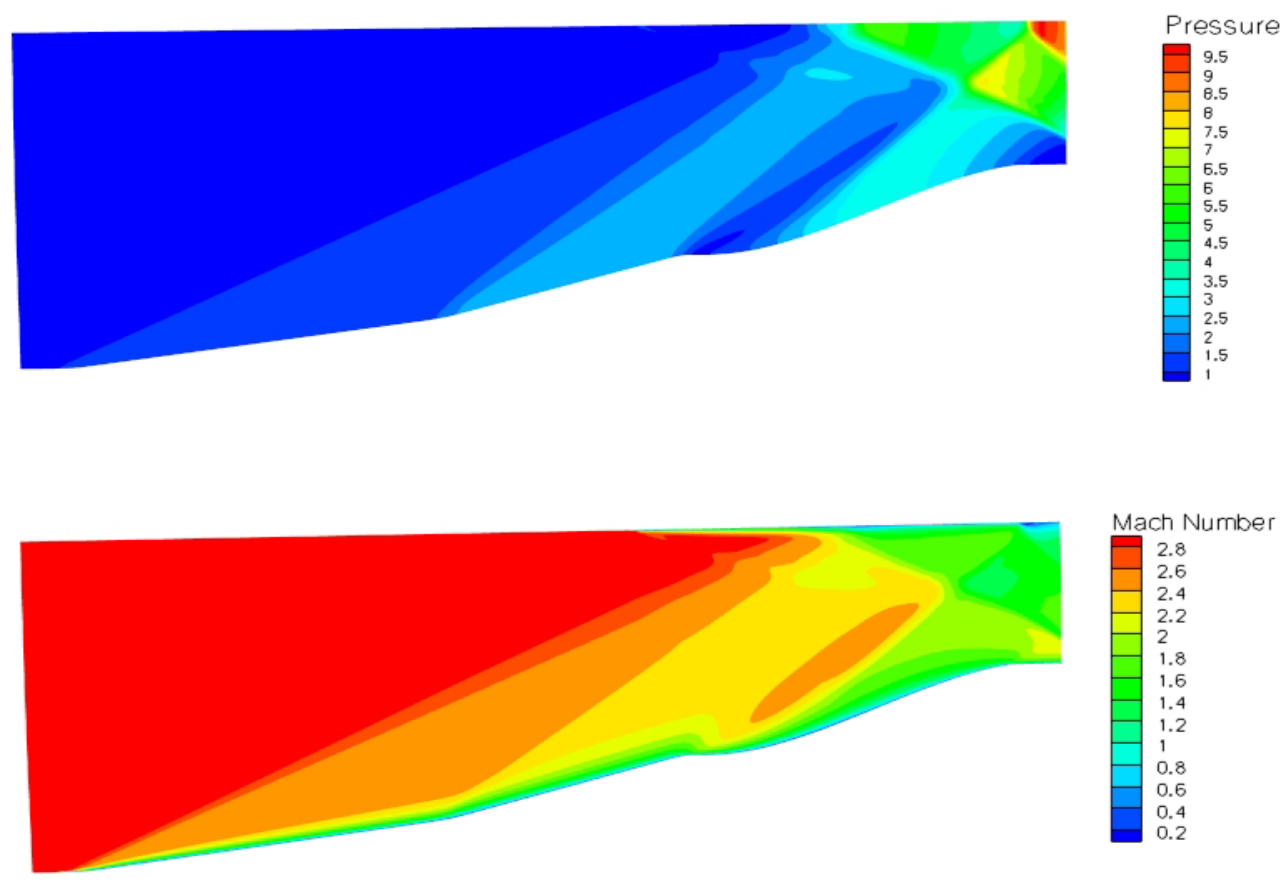

Figure 29: Static pressure and Mach number contours of a competitive inlet in Case 4. 

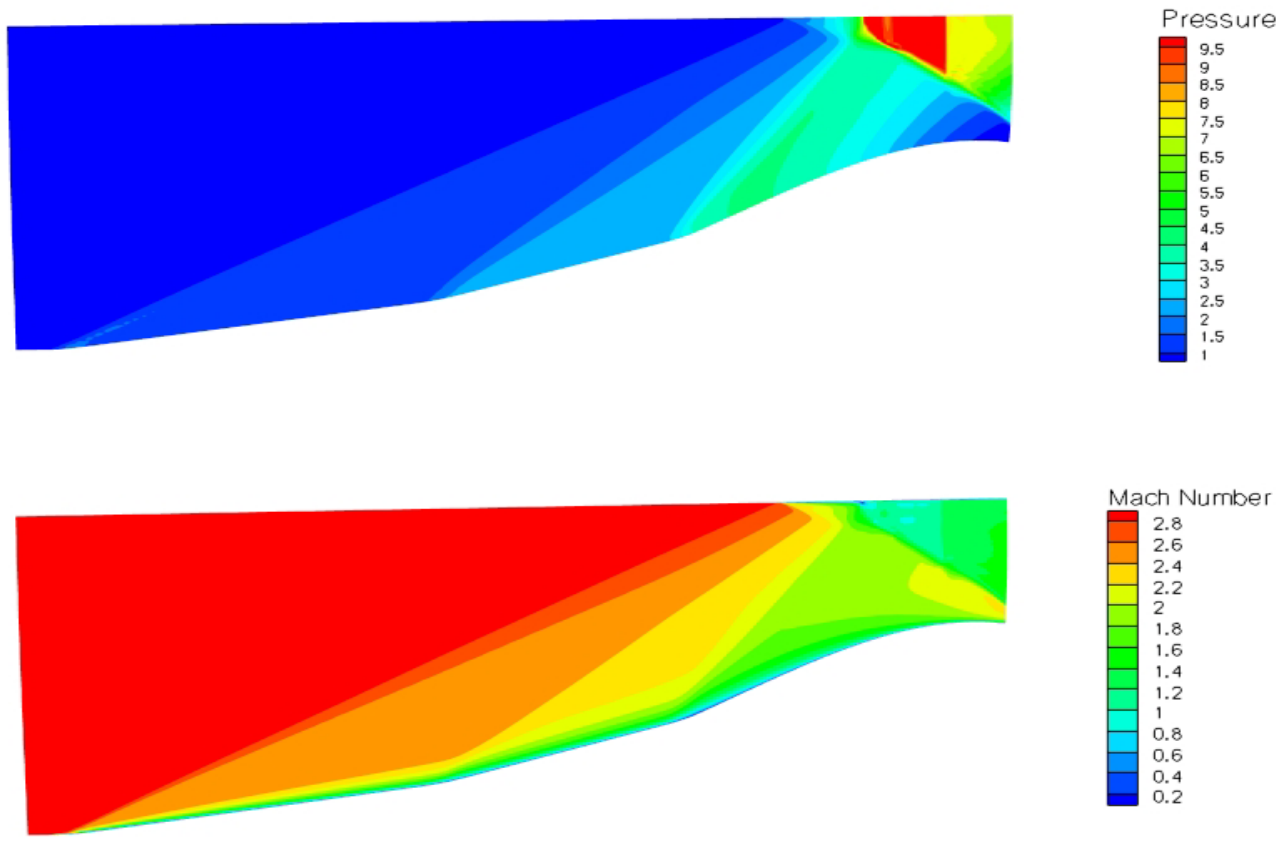

Figure 30: Static pressure and Mach number contours of the inlet with an unconventional geometric shape.

\section{Case 6: moveable cowl shaped with cubic polynomial function and fixed geometric shape of lower inlet surface}

Similar to case 5 , case 6 focuses on what an optimum cowl shape is. Again, a cubic polynomial function with coefficients of $\mathrm{a}, \mathrm{b}$ and $\mathrm{c}$ is used to describe the shape of cowl. They are three selected geometric design variables. The fourth variable is the location of the cowl leading edge. Those optimum values of the lower ramp surface obtained in case 5 are used in this case. Therefore, in this four design variables problem, the final point from GA is plotted in Figure 31. The blown section in the Mach number contours shows the geometric information on where lip starts and what the optimum cowl shape is, with imposed constraints of

$$
-2^{\circ} \leq \mathrm{A} \tan (\mathrm{a}) \geq 8^{\circ} ; \quad 1.2 \leq \mathrm{X}_{\text {cowl }} \geq 2.5 ; \quad 0.8 \leq \mathrm{H}_{\text {cowl }} \geq 1.15 .
$$

The first constraint is to confine the wedge angle of the cowl ramp between $-2^{\circ}$ and $8^{\circ}$ which is measured clockwise from the dotted reference line in Figure 31, while the third constraint limits the height of cowl ramp, $\mathrm{H}_{\text {cowl }}$, from the leading edge of the lower ramp surface. The second constraint on the location of cowl leading edge, $\mathrm{X}_{\text {cowl, }}$ is intended to confine the GA search within a smaller region. These three constraints are meant to limit the search domain in physical space and shorten the required time in GA run. Also, a bit of sensitivity test on population size is performed. Figure 31 presents the GA results with 30 individuals used, with a final total pressure recovery value of 0.676 . The cowl lip intersects with the horizontal line in a small angle of $3.148^{\circ}$ and shapes slightly concaved away from the lower ramp. Figure 32 shows results from 40 individuals, with cowl lip angled outwards from the ramp at $5.427^{\circ}$ and a very small region of flow separation near the end of curvature. Table 2 lists the values of these two sets of four design variables with their corresponding optimum total pressure recovery. Basically, Figures 31 and 32 present similar flowfield, with slightly more compression near the end of curved cowl in Figure 32. The forty individuals one has the optimum total pressure recovery being 0.678. GA may perform better if a larger pool of population is provided here. But, it comes with a higher demanding in computing resources. However, the improvement in performance is insignificant for this case, as shown in Table 2.

The major part of time taken is in the mesh generation and high fidelity computation in each generation in GA. For example, the averaged time for two-design-variable and 20 individuals case is about 20 seconds if ADD code is used. If Gridgen (C) is used, it takes a bit longer, 25 seconds, to complete Gridgen's elliptic solver which is set at 7500 iterations. An ordinary two design variables, single objective design case will take 50 (default stall number) to 70 generation, or 6 to 8.5 hours in average to complete. The processor used is the AMD Opteron ${ }^{\circledR}$, with i/o bus frequency of $1000 \mathrm{MHZ}$. 


\begin{tabular}{|l|l|l|l|l|l|}
\hline $\begin{array}{l}\text { \# of } \\
\text { individuals }\end{array}$ & $\mathrm{a}$ & $\mathrm{b}$ & $\mathrm{c}$ & $\mathrm{X}_{\text {cowl }}$ & Optimum total pressure recovery \\
\hline 30 & 0.055 & -0.222 & 0.203 & 2.106 & 0.676 \\
\hline 40 & 0.095 & -0.264 & 0.222 & 2.061 & 0.678 \\
\hline
\end{tabular}

Table 2: Sensitivity of population size on GA optimum values; results are from design problem of Case 6.

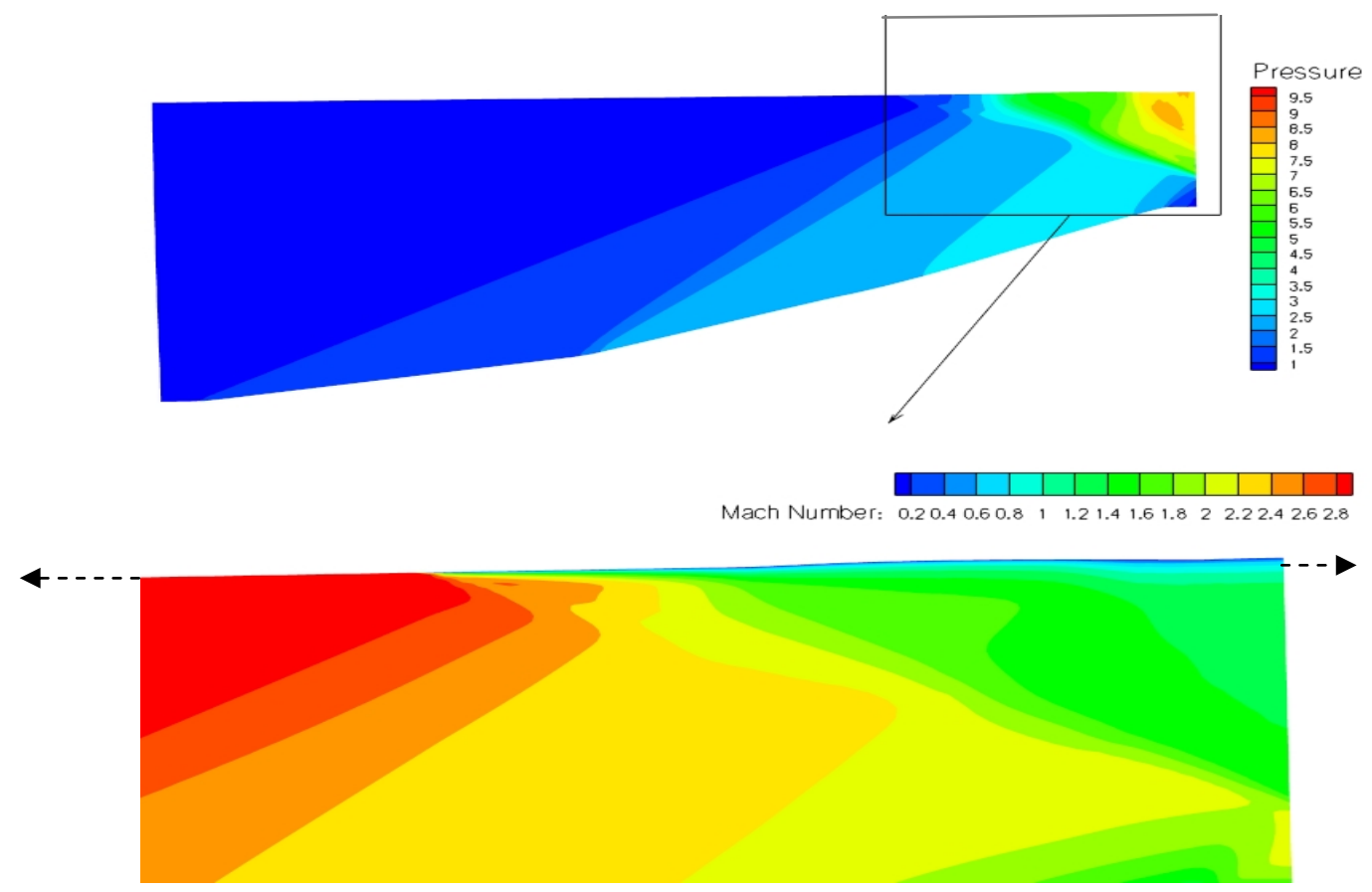

Figure 31: Static pressure and Mach number contours of results from thirty individuals in Case 6.
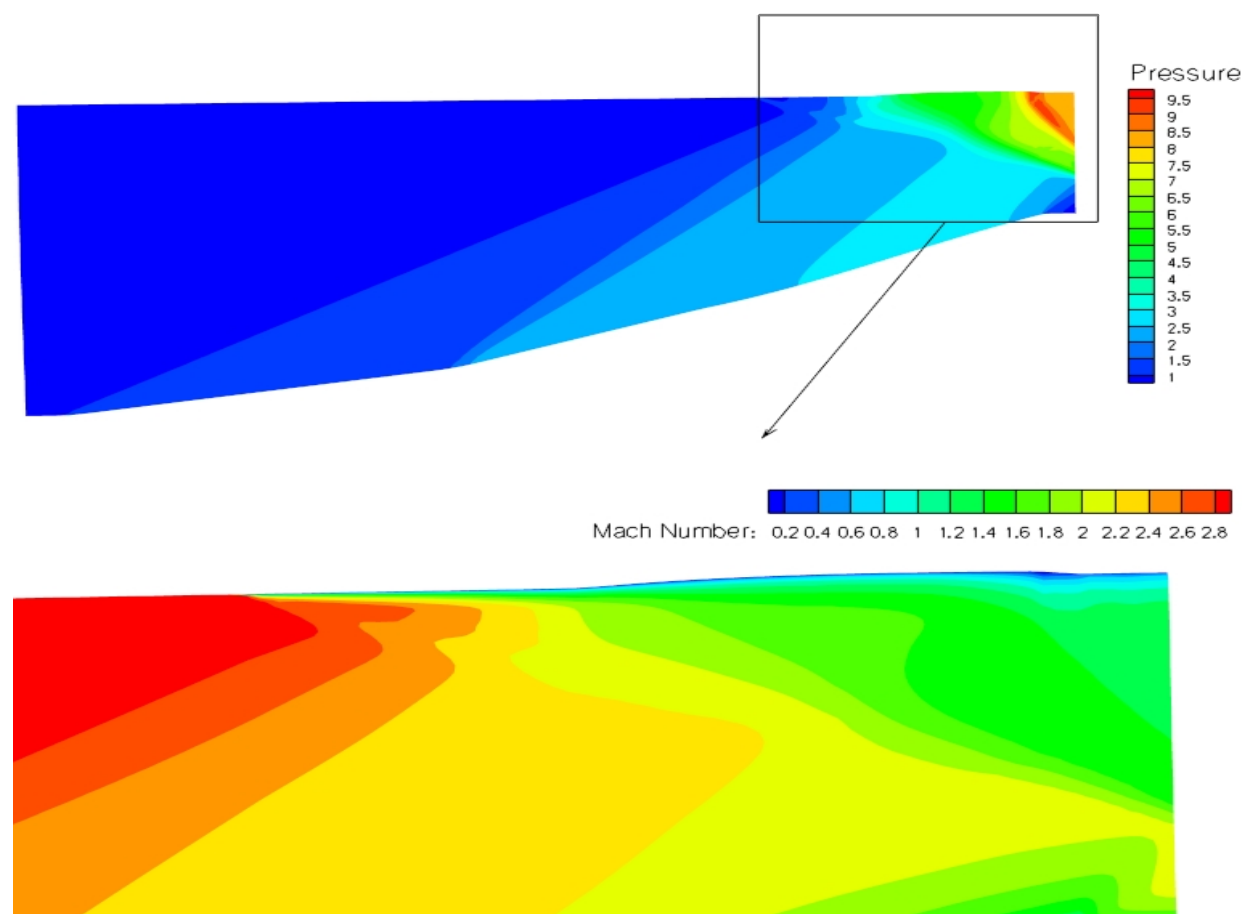

Figure 32: Static pressure and Mach number contours of results from forty individuals in Case 6. 


\section{Conclusion}

A practical inlet involves many compromises. The design process has always been very tedious and labor intensive, and could take weeks or months. In this paper, the feasibility of engaging genetic algorithm in inlet design is demonstrated. Results presented in this paper show this system can handle geometric design well in less than half a day, on a single processor, and without human intervention. Currently, up to four design variables are employed. The IPODS is demonstrated with products of unconventional, interesting geometry from GA and its automation capability makes the simulation- based design more efficient in terms of time and cost. The validity of the optimal design relies on the used design tool, like CFD. By no means, the showcase presented here is the best, nor the unique design. While this system can show promising best results under the definition of problem or sometimes can have difficulty in finding optimum, it needs design engineers to perform further analyses, for example, running experiments, redefining the design problem or using different options in optimization algorithm.

\section{Future plan}

Most high speed inlets require bleeding some percentage of inlet flow to stabilize the normal shock and control boundary layer separation due to flow disturbances, including atmospheric and engine-borne ones. The optimum amount of bleed is difficult to estimate and traditionally determined by experimental data. IPODS has a built-in bleed model which can be applied together with GA to optimize bleed in inlet.

\section{Acknowledgments}

Special thanks go to Dr. Ali Merchant at CADNexus Inc. for his technical assistance in integrating CAPRI into IPODS. This work is supported by Hypersonic Project, NASA Fundamental Aeronautics Program.

\section{Reference}

${ }^{1}$ Benson, T., Liou, M-F, Jones, W., and Trefny, C., "Nearly Interactive Parabolized Navier-Stokes Solver for High Speed Forebody and Inlet Flows," 47th AIAA Aerospace Science Meeting, Orlando, FL. January, 2009.

${ }^{2}$ CAPRI V3.08 User Documentation, CADNexus Inc, 2008.

${ }^{3}$ Anderson, O.L., "User's Manual for a Finite-Difference Calculation of Turbulent Swirling Compressible Flow Axisymmetric Ducts with Struts and Slot Cooled Walls,” USAAMRDL-TR-74-50, Vol. I, 1974.

${ }^{4}$ Glyph Reference Manual, Gridgen, Pointwise Inc.

${ }^{5}$ Genetic Algorithm and Direct Search Toolbox User's Guide, MathWorks Inc.

${ }^{6}$ Sedon, J., and E.L., Goldsmith, Intake Aerodynamics, AIAA Education Series, 1985, pp125.

7 Connors, J.F. And Meyer, R.C, "Design criteria for Axisymmetric and two-dimensional supersonic inlets and exits," NACA TN. Note 3589, 1956.

${ }^{8}$ Ran, H. and Mavris, D., "Preliminary Design of a 2D Supersonic Inlet to maximize Total Pressure Recovery," AIAA $5^{\text {th }}$ Aviation, Technology, Integration, and Operations Conference, Arlington, VA, September, 2005.

${ }^{9}$ McDonald, H and Camarata, F.J., "An Extended Mixing Length Approach for Computing Turbulent Boundary Layer Development," Proceeding of the Stanford Conference on Computation of Turbulent Boundary Layers, Vol. I, Stanford University, 1969, pp 83-94. 